\title{
Computing Time-Dependent Bid Prices in Network Revenue Management Problems
}

\author{
Sumit Kunnumkal \\ Indian School of Business, Gachibowli, Hyderabad, 500032, India \\ sumit_kunnumkal@isb.edu \\ Huseyin Topaloglu \\ School of Operations Research and Information Engineering, \\ Cornell University, Ithaca, New York 14853, USA \\ topaloglu@orie.cornell.edu
}

July 16, 2009

\begin{abstract}
We propose a new method to compute bid prices in network revenue management problems. The novel aspect of our method is that it naturally provides dynamic bid prices that depend on how much time is left until departure. We show that our method provides an upper bound on the optimal total expected revenue and this upper bound is tighter than the one provided by the widely known deterministic linear programming approach. Furthermore, it is possible to use the bid prices computed by our method as a starting point in a dynamic programming decomposition-like idea to decompose the network revenue management problem by the flight legs and to obtain dynamic and capacity-dependent bid prices. Our computational experiments indicate that the proposed method improves on many standard benchmarks.
\end{abstract}


The idea of bid prices forms a powerful tool for building good and practical policies for network revenue management problems. This idea associates a bid price with each flight leg that captures the opportunity cost of a unit of capacity. An itinerary request is accepted only when there is enough capacity and the revenue from the itinerary request exceeds the sum of the bid prices associated with the flight legs that are in the requested itinerary; see Williamson (1992) and Talluri and van Ryzin (1998). Although it is known that the optimal policies are not necessarily characterized by bid prices, the intuitive appeal and ease of implementation of the bid price policies make them a popular choice in practice.

One of the traditional approaches for computing bid prices is based on solving a deterministic linear program. This linear program can be viewed as a deterministic approximation to the network revenue management problem that is formulated under the assumption that the numbers of itinerary requests are known in advance and they take on their expected values. In the deterministic linear program, there exists one constraint for each flight leg and the right sides of these constraints are the remaining leg capacities. Therefore, the optimal values of the dual variables associated with these capacity constraints are used as bid prices. Nevertheless, since the deterministic linear program assumes that the numbers of itinerary requests are known in advance, it does not accurately capture the temporal dynamics of the arrivals of the itinerary requests.

In this paper, we propose a new method to compute dynamic bid prices that depend on how much time is left until the time of departure. Our goal is to compute bid prices that capture the temporal dynamics of the arrivals of the itinerary requests somewhat more accurately than the deterministic linear program. The linear program described above can be viewed as a deterministic approximation, whereas our method directly works with the dynamic programming formulation of the network revenue management problem. The main idea behind our method is to relax the capacity constraints in the dynamic programming formulation by associating Lagrange multipliers with them. In this case, the optimality equation decomposes by the time periods and we obtain a simple expression for the value function. We show that a good set of values for the Lagrange multipliers can be obtained by solving a linear program. Since our Lagrange multipliers depend on how much time is left until departure, the bid prices that we obtain also depend on how much time is left until departure.

Our method shares many of the appealing features of the traditional linear programming approach for computing bid prices. To begin with, it is known that the optimal objective value of the deterministic linear program provides an upper bound on the optimal total expected revenue and we show that our method also provides such an upper bound. Furthermore, a popular method to compute dynamic bid prices is based on decomposing the network revenue management problem into a sequence of single leg revenue management problems. This idea is known as dynamic programming decomposition and the bid prices computed by the deterministic linear program serve as a starting point for this method; see Section 3.4.4 in Talluri and van Ryzin (2004). As a matter of fact, it is possible to show that dynamic programming decomposition computes dynamic and capacity-dependent bid prices that depend not only on how much time is left until departure, but also on how many units of capacity are left on the flight legs. We show that the bid prices computed by our method can also be used in a dynamic programming decomposition-like idea to compute dynamic and capacity-dependent bid prices. Finally, 
Talluri and van Ryzin (1998) show that the bid prices computed by the deterministic linear program are asymptotically optimal as the capacities on the flight legs and the expected numbers of itinerary requests increase linearly with the same rate. It is possible to show that the bid prices computed by our method are also asymptotically optimal in the same sense. We do not pursue this result here, but a related technical report provides the details; see Topaloglu and Kunnumkal (2006). In addition to these parallels between our method and the deterministic linear program, we show that the upper bound on the optimal total expected revenue provided by our method is tighter than the one provided by the linear program. Furthermore, computational experiments indicate that the bid prices computed by our method can perform significantly better than those computed by the deterministic linear program.

Network revenue management is an active area of research. Simpson (1989) and Williamson (1992) were the first to use the deterministic linear program to compute bid prices. Talluri and van Ryzin (1998) give a careful study of bid price policies and show the asymptotic optimality result mentioned above for the bid prices computed by the deterministic linear program. Talluri and van Ryzin (1999) propose a randomized version of the deterministic linear program that uses samples of the numbers of the itinerary requests and this randomization significantly improves the performance. Their goal is to alleviate the shortcoming that the deterministic linear program uses only the expected numbers of the itinerary requests and does not pay attention to the probability distributions. Bertsimas and Popescu (2003) compute bid prices by using the change in the optimal objective value of the deterministic linear program induced by a change in the right sides of certain constraints. By doing so, they try to capture the total opportunity cost of the leg capacities consumed by an itinerary request more accurately. In their paper, they also show how to extend the deterministic linear program to handle cancellations and no shows. Section 3.4.4 in Talluri and van Ryzin (2004) gives a description of the dynamic programming decomposition idea that can be used to decompose the network revenue management problem into a sequence of single leg revenue management problems so as to compute dynamic and capacity-dependent bid prices. As mentioned above, the bid prices computed by the deterministic linear program play an important role in dynamic programming decomposition.

Two papers in the literature are particularly related to our work. First, Adelman (2007) uses linear approximations to the value functions in the dynamic programming formulation of the network revenue management problem. To choose the slope parameters of these approximations, he plugs them into a linear program that represents the dynamic programming formulation of the problem. The number of constraints in this linear program is exponential in the number of flight legs, but the number of decision variables is manageable and he solves this linear program by using column generation on its dual. Similar to our dynamic bid prices, the policy obtained from his linear value function approximations turns out to be a dynamic bid price policy. Second, Topaloglu (2009) computes bid prices by decomposing the network revenue management problem into a sequence of single leg revenue management problems. He uses a Lagrangian relaxation idea, but he does not relax the capacity constraints like we do. Instead, he observes that if an itinerary request is accepted, then the capacities on all of the flight legs that are in this itinerary have to be consumed. He relaxes this requirement so that it becomes allowable to individually accept or reject the capacities on the flight legs that are in the requested itinerary. Under this relaxation, the network revenue management problem decomposes by the flight legs. 
In our demand model, we assume that each customer arrives into the system with the intention of purchasing one particular itinerary and the decision maker decides whether to accept or reject this itinerary request. This is known as the independent demand model. In reality, there may be many different itineraries that are acceptable to a customer. In this case, the decision maker decides which group of itineraries to make available for purchase. Each customer observes the group of itineraries available for purchase and makes a choice. There has been a lot of recent attention in the literature towards modeling this kind of customer choice behavior. It turns out that many of the models that work for the independent demand setting can be extended to the customer choice setting. Gallego, Iyengar, Phillips and Dubey (2004) and Liu and van Ryzin (2008) extend the deterministic linear program, Zhang and Adelman (2006) extend the linear value function approximations of Adelman (2007) and Kunnumkal and Topaloglu (2007) extend the decomposition strategy of Topaloglu (2009). The method that we develop in this paper can also be extended to the customer choice setting and we pursue this extension in Kunnumkal and Topaloglu (2008b).

Our main methodological machinery is based on relaxing the complicating constraints in a dynamic program by associating Lagrange multipliers with them. The connections between the duality and control theory appear in the earlier literature; see Varaiya (1998) and Bertsekas (2001). Recently, Hawkins (2003) and Adelman and Mersereau (2008) revive these connections by studying dynamic programs that would decompose by the components of the state variable if a few constraints did not play a linking role. They call these dynamic programs as weakly coupled and show that such dynamic programs are particularly suitable for Lagrangian relaxation ideas. Karmarkar (1981), Cheung and Powell (1996), Castanon (1997) and Kunnumkal and Topaloglu (2008a) apply Lagrangian relaxation ideas to the dynamic programs arising in multi-location inventory allocation, fleet management, sensor management and multi-echelon inventory distribution settings.

We make the following research contributions in this paper. 1) We propose a new method to compute dynamic bid prices that depend on how much time is left until departure. 2) We show that our method provides an upper bound on the optimal total expected revenue and this upper bound is tighter than the one provided by the deterministic linear program. 3) Our method is based on relaxing the capacity constraints by associating Lagrange multipliers with them. We show that a good set of Lagrange multipliers can be obtained by solving a linear program. 4) We show that the bid prices computed by our method can be used in a dynamic programming decomposition-like idea to compute dynamic and capacity-dependent bid prices. 5) Computational experiments indicate that the bid prices computed by our method perform noticeably better than those computed by many standard benchmark methods.

The rest of the paper is organized as follows. In Section 1, we formulate the network revenue management problem as a dynamic program. In Section 2, we present the basic Lagrangian relaxation idea. In Section 3, we show that a good set of values for the Lagrange multipliers can be obtained by solving a linear program. In Section 4, we show how to use the bid prices computed by our method as a starting point to decompose the network revenue management problem by the flight legs and to compute dynamic and capacity-dependent bid prices. In Section 5, we contrast our method with other approaches for computing bid prices. In Section 6, we present computational experiments. 


\section{Problem Formulation}

We have a set of flight legs that can be used to satisfy the itinerary requests that arrive randomly over time. At each time period, an itinerary request arrives and we have to decide whether to accept or reject the itinerary request. An accepted itinerary request generates a revenue and consumes the capacities on the relevant flight legs. A rejected itinerary request simply leaves the system.

The problem takes place over the finite planning horizon $\mathcal{T}=\{1, \ldots, \tau\}$ and all flight legs depart at time period $\tau+1$. The set of flight legs is $\mathcal{L}$ and the set of itineraries is $\mathcal{J}$. The capacity on flight $\operatorname{leg} i$ is $c_{i}$. If we accept a request for itinerary $j$, then we generate a revenue of $f_{j}$ and consume $a_{i j}$ units of capacity on flight leg $i$. We naturally have $a_{i j}=0$ when itinerary $j$ does not use flight leg $i$. The probability that a request for itinerary $j$ arrives at time period $t$ is $p_{j t}$. For notational brevity, we assume that $\sum_{j \in \mathcal{J}} p_{j t}=1$ for all $t \in \mathcal{T}$ so that there is an itinerary request at every time period with probability 1 . If there is a strictly positive probability that no itinerary requests arrive at certain time periods, then we can cover this case simply by defining a fictitious itinerary $\psi$ with $f_{\psi}=0, a_{i \psi}=0$ for

all $i \in \mathcal{L}$ and $p_{\psi t}=1-\sum_{j \in \mathcal{J}} p_{j t}$ for all $t \in \mathcal{T}$. We assume that the itinerary requests at different time periods are independent.

We let $x_{i t}$ be the remaining capacity on flight leg $i$ at time period $t$ so that $x_{t}=\left\{x_{i t}: i \in \mathcal{L}\right\}$ captures the remaining leg capacities at time period $t$. We capture the decisions at time period $t$ by $u_{t}=\left\{u_{j t}: j \in \mathcal{J}\right\}$, where $u_{j t}$ takes value 1 if we accept a request for itinerary $j$ at time period $t$ and $u_{j t}$ takes value 0 if we reject a request for itinerary $j$ at time period $t$. In this case, the set of feasible decisions is given by

$$
\mathcal{U}\left(x_{t}\right)=\left\{u_{t} \in\{0,1\}^{|\mathcal{J}|}: a_{i j} u_{j t} \leq x_{i t} \quad \forall i \in \mathcal{L}, j \in \mathcal{J}\right\} .
$$

These constraints ensure that if we accept a request for itinerary $j$ at time period $t$, then the capacity consumed by itinerary $j$ on flight leg $i$ does not exceed the remaining capacity on flight leg $i$.

Using $x_{t}$ as the state variable and letting $e_{i}$ be the $|\mathcal{L}|$-dimensional unit vector with a 1 in the element corresponding to $i \in \mathcal{L}$, the optimal policy can be found by computing the value functions through the optimality equation

$$
V_{t}\left(x_{t}\right)=\max _{u_{t} \in \mathcal{U}\left(x_{t}\right)}\left\{\sum_{j \in \mathcal{J}} p_{j t}\left[f_{j} u_{j t}+V_{t+1}\left(x_{t}-u_{j t} \sum_{i \in \mathcal{L}} a_{i j} e_{i}\right)\right]\right\} .
$$

As a function of the state variable $x_{t}$, it is easy to see that the optimal decisions at time period $t$ are given by $\hat{u}_{t}\left(x_{t}\right)=\left\{\hat{u}_{j t}\left(x_{t}\right): j \in \mathcal{J}\right\}$, where

$$
\hat{u}_{j t}\left(x_{t}\right)= \begin{cases}1 & \text { if } f_{j}+V_{t+1}\left(x_{t}-\sum_{i \in \mathcal{L}} a_{i j} e_{i}\right) \geq V_{t+1}\left(x_{t}\right) \text { and } a_{i j} \leq x_{i t} \text { for all } i \in \mathcal{L} \\ 0 & \text { otherwise. }\end{cases}
$$

A complicating factor in the optimality equation in (1) is the constraints $a_{i j} u_{j t} \leq x_{i t}$ for all $i \in \mathcal{L}$, $j \in \mathcal{J}$ captured by the feasible set $\mathcal{U}\left(x_{t}\right)$. In particular, if these constraints did not exist, then the optimality equation in (1) would decompose by the time periods. This suggests relaxing the constraints $a_{i j} u_{j t} \leq x_{i t}$ for all $i \in \mathcal{L}, j \in \mathcal{J}$ by associating Lagrange multipliers with them, in which case the optimality equation in (1) has a simple solution. We build on this idea in the next section. 


\section{Lagrangian Relaxation Strategy}

We propose relaxing the constraints $a_{i j} u_{j t} \leq x_{i t}$ for all $i \in \mathcal{L}, j \in \mathcal{J}$ in the optimality equation in (1) by associating Lagrange multipliers with them. In particular, we associate the nonnegative Lagrange multipliers $\left\{\alpha_{i j t}: i \in \mathcal{L}, j \in \mathcal{J}\right\}$ with the constraints $a_{i j} u_{j t} \leq x_{i t}$ for all $i \in \mathcal{L}, j \in \mathcal{J}$ and solve the optimality equation

$$
V_{t}^{\alpha}\left(x_{t}\right)=\max _{u_{t} \in\{0,1\} \mid \mathcal{J \mathcal { J }}}\left\{\sum_{j \in \mathcal{J}} p_{j t}\left\{\left[f_{j}-\sum_{i \in \mathcal{L}} a_{i j} \alpha_{i j t}\right] u_{j t}+\sum_{i \in \mathcal{L}} \alpha_{i j t} x_{i t}+V_{t+1}^{\alpha}\left(x_{t}-u_{j t} \sum_{i \in \mathcal{L}} a_{i j} e_{i}\right)\right\}\right\} .
$$

We note that the Lagrange multipliers above are scaled by $\left\{p_{j t}: j \in \mathcal{J}\right\}$ for notational brevity. If we have $p_{j t}=0$, then the Lagrange multipliers $\left\{\alpha_{i j t}: i \in \mathcal{L}\right\}$ are inconsequential and scaling the Lagrange multipliers in this fashion does not create a complication. We use the superscript $\alpha=\left\{\alpha_{i j t}: i \in\right.$ $\mathcal{L}, j \in \mathcal{J}, t \in \mathcal{T}\}$ in the value functions to emphasize that the solution to the optimality equation in (3) depends on the Lagrange multipliers. Since the Lagrange multiplier $\alpha_{i j t}$ is associated with the capacity availability constraint for flight leg $i$ and itinerary $j$ at time period $t$, it roughly captures the opportunity cost of a unit of capacity on flight leg $i$ given that there is a request for itinerary $j$ at time period $t$. As we shortly demonstrate, $\alpha_{i j t}$ is ultimately related to the bid price associated with flight $\operatorname{leg} i$ at time period $t$.

The next proposition shows that there is a simple solution to the optimality equation in (3). In the next proposition and throughout the rest of the paper, we let

$$
\begin{gathered}
r_{i t}^{\alpha}=\sum_{j \in \mathcal{J}} p_{j t} \alpha_{i j t}+\ldots+\sum_{j \in \mathcal{J}} p_{j \tau} \alpha_{i j \tau} \\
L_{j t}^{\alpha}=\left[f_{j}-\sum_{i \in \mathcal{L}} a_{i j} \alpha_{i j t}-\sum_{i \in \mathcal{L}} a_{i j} r_{i, t+1}^{\alpha}\right]^{+},
\end{gathered}
$$

where we use $[\cdot]^{+}=\max \{0, \cdot\}$ and follow the convention that $r_{i, \tau+1}^{\alpha}=0$. We emphasize that both $r_{i t}^{\alpha}$ and $L_{j t}^{\alpha}$ are simple functions of the Lagrange multipliers. Furthermore, noting the interpretation of $\alpha_{i j t}$ above, $r_{i t}^{\alpha}$ roughly captures the total expected opportunity cost of a unit of capacity on flight leg $i$ over time periods $\{t, \ldots, \tau\}$.

Proposition 1 For all $t \in \mathcal{T}$, we have

$$
V_{t}^{\alpha}\left(x_{t}\right)=\sum_{i \in \mathcal{L}} r_{i t}^{\alpha} x_{i t}+\sum_{j \in \mathcal{J}} p_{j t} L_{j t}^{\alpha}+\ldots+\sum_{j \in \mathcal{J}} p_{j \tau} L_{j \tau}^{\alpha}
$$

Proof We show the result by induction over the time periods. It is easy to show the result for the last time period. Assuming that the result holds for time period $t+1$, (3) implies that

$$
\begin{aligned}
V_{t}^{\alpha}\left(x_{t}\right)=\max _{u_{t} \in\{0,1\}|\mathcal{J}|}\left\{\sum _ { j \in \mathcal { J } } p _ { j t } \left\{\left[f_{j}-\sum_{i \in \mathcal{L}} a_{i j} \alpha_{i j t}\right] u_{j t}+\sum_{i \in \mathcal{L}} \alpha_{i j t} x_{i t}\right.\right. & \left.\left.+\sum_{i \in \mathcal{L}} r_{i, t+1}^{\alpha}\left[x_{i t}-a_{i j} u_{j t}\right]\right\}\right\} \\
& +\sum_{j \in \mathcal{J}} p_{j, t+1} L_{j, t+1}^{\alpha}+\ldots+\sum_{j \in \mathcal{J}} p_{j \tau} L_{j \tau}^{\alpha} .
\end{aligned}
$$


Arranging the terms and letting $\mathbf{1}(\cdot)$ be the indicator function, the optimal values of the decision variables $\left\{u_{j t}: j \in \mathcal{J}\right\}$ in the problem above are $\left\{\mathbf{1}\left(f_{j}-\sum_{i \in \mathcal{L}} a_{i j} \alpha_{i j t}-\sum_{i \in \mathcal{L}} a_{i j} r_{i, t+1}^{\alpha} \geq 0\right): j \in \mathcal{J}\right\}$ and the result follows from the definition of $L_{j t}^{\alpha}$ and the fact that $r_{i t}^{\alpha}=\sum_{j \in \mathcal{J}} p_{j t} \alpha_{i j t}+r_{i, t+1}^{\alpha}$.

Therefore, the value functions computed through the optimality equation in (3) are linear functions of the remaining leg capacities. This is one of the connections between our method and the method proposed by Adelman (2007), as both methods end up using linear value function approximations. In Section 5, we show a result that indicates that the method proposed by Adelman (2007) is potentially stronger in terms of the tightness of the upper bounds. Nevertheless, our computational experiments in Section 6 demonstrate that the upper bounds obtained by the two methods are identical for all of our test problems. Kunnumkal and Topaloglu (2008b) extend Proposition 1 to the customer choice setting, but the computation of $L_{j t}^{\alpha}$ is significantly more complicated in their case.

Assuming that the value functions $\left\{V_{t}^{\alpha}(\cdot): t \in \mathcal{T}\right\}$ computed through the optimality equation in (3) are good approximations to the value functions $\left\{V_{t}(\cdot): t \in \mathcal{T}\right\}$ computed through the optimality equation in (1), we propose making the itinerary acceptance decisions by replacing $\left\{V_{t}(\cdot): t \in \mathcal{T}\right\}$ on the right side of $(2)$ with $\left\{V_{t}^{\alpha}(\cdot): t \in \mathcal{T}\right\}$. We shortly dwell on the questions of what we mean by good approximations and how we can choose the Lagrange multipliers so that $\left\{V_{t}^{\alpha}(\cdot): t \in \mathcal{T}\right\}$ are good approximations to $\left\{V_{t}(\cdot): t \in \mathcal{T}\right\}$. Leaving these questions aside for the time being, the idea of approximating $\left\{V_{t}(\cdot): t \in \mathcal{T}\right\}$ in $(2)$ by $\left\{V_{t}^{\alpha}(\cdot): t \in \mathcal{T}\right\}$ implies that if we have

$$
f_{j}+V_{t+1}^{\alpha}\left(x_{t}-\sum_{i \in \mathcal{L}} a_{i j} e_{i}\right) \geq V_{t+1}^{\alpha}\left(x_{t}\right)
$$

and $a_{i j} \leq x_{i t}$ for all $i \in \mathcal{L}$, then we accept a request for itinerary $j$ at time period $t$. Otherwise, we reject the itinerary request. Since we have $V_{t+1}^{\alpha}\left(x_{t}\right)-V_{t+1}^{\alpha}\left(x_{t}-\sum_{i \in \mathcal{L}} a_{i j} e_{i}\right)=\sum_{i \in \mathcal{L}} a_{i j} r_{i, t+1}^{\alpha}$ by $(4)$, this idea is equivalent to accepting a request for itinerary $j$ at time period $t$ when we have

$$
f_{j} \geq \sum_{i \in \mathcal{L}} a_{i j} r_{i, t+1}^{\alpha}
$$

and $a_{i j} \leq x_{i t}$ for all $i \in \mathcal{L}$. Therefore, we can view $r_{i, t+1}^{\alpha}$ as the bid price associated with flight leg $i$ at time period $t$. Similar to the idea of bid prices described in the introduction, if there is enough capacity and the revenue from an itinerary request exceeds the sum of the bid prices associated with the flight legs that are in the requested itinerary, then we accept the itinerary request. It is important to note that the bid price associated with a flight leg in (5) depends on how much time is left until departure. In particular, since the Lagrange multipliers are nonnegative and $r_{i t}^{\alpha}=\sum_{j \in \mathcal{J}} p_{j t} \alpha_{i j t}+r_{i, t+1}^{\alpha}$, we have $r_{i t}^{\alpha} \geq r_{i, t+1}^{\alpha} \geq \ldots \geq r_{i, \tau+1}^{\alpha}$ and the bid price associated with flight leg $i$ decreases as we approach the departure time of the flight legs. This is in agreement with the intuitive expectation that the opportunity cost of a unit of capacity should decrease as we approach the departure time and fewer opportunities to utilize the leg capacities remain.

\section{Choosing the Lagrange Multipliers}

In this section, we consider the question of how to choose the Lagrange multipliers. We begin with the next proposition, which shows that we can obtain upper bounds on the value functions by solving the optimality equation in (3). 
Proposition 2 If the Lagrange multipliers are nonnegative, then we have $V_{t}\left(x_{t}\right) \leq V_{t}^{\alpha}\left(x_{t}\right)$ for all $t \in \mathcal{T}$.

Proof We show the result by induction over the time periods. It is easy to show the result for the last time period. Assuming that the result holds for time period $t+1$ and letting $\hat{u}_{t}=\left\{\hat{u}_{j t}: j \in \mathcal{J}\right\}$ be an optimal solution to problem (1), we have

$$
\begin{aligned}
& V_{t}\left(x_{t}\right)=\sum_{j \in \mathcal{J}} p_{j t} {\left[f_{j} \hat{u}_{j t}+V_{t+1}\left(x_{t}-\hat{u}_{j t} \sum_{i \in \mathcal{L}} a_{i j} e_{i}\right)\right] } \\
& \leq \sum_{j \in \mathcal{J}} p_{j t}\left\{\left[f_{j}-\sum_{i \in \mathcal{L}} a_{i j} \alpha_{i j t}\right] \hat{u}_{j t}+\sum_{i \in \mathcal{L}} \alpha_{i j t} x_{i t}+V_{t+1}^{\alpha}\left(x_{t}-\hat{u}_{j t} \sum_{i \in \mathcal{L}} a_{i j} e_{i}\right)\right\} \leq V_{t}^{\alpha}\left(x_{t}\right),
\end{aligned}
$$

where the first inequality follows from the induction assumption and the fact that $\alpha_{i j t} \geq 0$ and $a_{i j} \hat{u}_{j t} \leq$ $x_{i t}$ for all $i \in \mathcal{L}, j \in \mathcal{J}$ and the second inequality follows from the fact that $\hat{u}_{t}$ is a feasible but not necessarily an optimal solution to problem (3).

Proposition 2 is a standard upper bound result that often arises in the applications of Lagrangian relaxation ideas. Cheung and Powell (1996) and Kunnumkal and Topaloglu (2008b) show similar results in fleet management and network revenue management with customer choice settings. Adelman and Mersereau (2008) show a similar result for weakly coupled dynamic programs over an infinite horizon, but their proof simplifies significantly when one considers the finite horizon case.

Since the initial leg capacities are given by $c=\left\{c_{i}: i \in \mathcal{L}\right\}$, the optimal total expected revenue over the planning horizon is $V_{1}(c)$. Proposition 2 implies that $V_{1}(c)$ is bounded from above by $V_{1}^{\alpha}(c)$ as long as the Lagrange multipliers are nonnegative. Therefore, to obtain the tightest possible upper bound on $V_{1}(c)$, we can solve the problem

$$
\min _{\alpha \geq 0}\left\{V_{1}^{\alpha}(c)\right\}
$$

It turns out that we can obtain an optimal solution to problem (6) by solving a linear program. To see this, we first note that

$$
V_{1}^{\alpha}(c)=\sum_{i \in \mathcal{L}} r_{i 1}^{\alpha} c_{i}+\sum_{t \in \mathcal{T}} \sum_{j \in \mathcal{J}} p_{j t} L_{j t}^{\alpha}
$$

by Proposition 1. In this case, the next proposition shows that the linear program

$$
\begin{array}{rlrl}
\min & \sum_{i \in \mathcal{L}} c_{i} \rho_{i 1}+\sum_{t \in \mathcal{T}} \sum_{j \in \mathcal{J}} p_{j t} \Gamma_{j t} & \\
\text { subject to } & \Gamma_{j t} \geq f_{j}-\sum_{i \in \mathcal{L}} a_{i j} \alpha_{i j t}-\sum_{i \in \mathcal{L}} a_{i j} \rho_{i, t+1} & & \forall j \in \mathcal{J}, t \in \mathcal{T} \backslash\{\tau\} \\
& \Gamma_{j \tau} \geq f_{j}-\sum_{i \in \mathcal{L}} a_{i j} \alpha_{i j \tau} & \forall j \in \mathcal{J} \\
\rho_{i t}=\sum_{j \in \mathcal{J}} p_{j t} \alpha_{i j t}+\ldots+\sum_{j \in \mathcal{J}} p_{j \tau} \alpha_{i j \tau} & \forall i \in \mathcal{L}, t \in \mathcal{T} \\
\rho_{i t} \text { is free, } \Gamma_{j t} \geq 0, \alpha_{i j t} \geq 0 & \forall i \in \mathcal{L}, j \in \mathcal{J}, t \in \mathcal{T}
\end{array}
$$

is equivalent to problem (6). In the next proposition and throughout the rest of the paper, we use $\hat{\zeta}$ to denote the optimal objective value of problem (8)-(12). 
Proposition 3 We have $\hat{\zeta}=\min _{\alpha \geq 0}\left\{V_{1}^{\alpha}(c)\right\}$.

Proof If $\hat{\alpha}=\left\{\hat{\alpha}_{i j t}: i \in \mathcal{L}, j \in \mathcal{J}, t \in \mathcal{T}\right\}$ is an optimal solution to problem (6), then the definitions of $r_{i t}^{\alpha}$ and $L_{j t}^{\alpha}$ imply that $\left\{r_{i t}^{\hat{\alpha}}: i \in \mathcal{L}, t \in \mathcal{T}\right\},\left\{L_{j t}^{\hat{\alpha}}: j \in \mathcal{J}, t \in \mathcal{T}\right\},\left\{\hat{\alpha}_{i j t}: i \in \mathcal{L}, j \in \mathcal{J}, t \in \mathcal{T}\right\}$ is a feasible solution to problem (8)-(12). In this case, (7) implies that we have $\hat{\zeta} \leq \sum_{i \in \mathcal{L}} c_{i} r_{i 1}^{\hat{\alpha}}+\sum_{t \in \mathcal{T}} \sum_{j \in \mathcal{J}} p_{j t} L_{j t}^{\hat{\alpha}}=$ $V_{1}^{\hat{\alpha}}(c)=\min _{\alpha \geq 0}\left\{V_{1}^{\alpha}(c)\right\}$.

On the other hand, if $\left\{\hat{\rho}_{i t}: i \in \mathcal{L}, t \in \mathcal{T}\right\},\left\{\hat{\Gamma}_{j t}: j \in \mathcal{J}, t \in \mathcal{T}\right\}, \hat{\alpha}=\left\{\hat{\alpha}_{i j t}: i \in \mathcal{L}, j \in \mathcal{J}, t \in \mathcal{T}\right\}$ is an optimal solution to problem (8)-(12), then we have $\hat{\rho}_{i t}=r_{i t}^{\hat{\alpha}}$ for all $i \in \mathcal{L}, t \in \mathcal{T}$ by constraints (11) and the definition of $r_{i t}^{\alpha}$. Constraints (9) and the fact that problem (8)-(12) is a minimization problem imply that $\hat{\Gamma}_{j t}=\left[f_{j}-\sum_{i \in \mathcal{L}} a_{i j} \hat{\alpha}_{i j t}-\sum_{i \in \mathcal{L}} a_{i j} \hat{\rho}_{i, t+1}\right]^{+}=\left[f_{j}-\sum_{i \in \mathcal{L}} a_{i j} \hat{\alpha}_{i j t}-\sum_{i \in \mathcal{L}} a_{i j} r_{i, t+1}^{\hat{\alpha}}\right]^{+}=L_{j t}^{\hat{\alpha}}$ for all $j \in$ $\mathcal{J}, t \in \mathcal{T} \backslash\{\tau\}$, where the last equality follows from the definition of $L_{j t}^{\alpha}$. Similarly, constraints (10) and the fact that problem (8)-(12) is a minimization problem imply that $\hat{\Gamma}_{j \tau}=\left[f_{j}-\sum_{i \in \mathcal{L}} a_{i j} \hat{\alpha}_{i j \tau}\right]^{+}=L_{j \tau}^{\hat{\alpha}}$ for all $j \in \mathcal{J}$. In this case, we have $\hat{\zeta}=\sum_{i \in \mathcal{L}} c_{i} \hat{\rho}_{i 1}+\sum_{t \in \mathcal{T}} \sum_{j \in \mathcal{J}} p_{j t} \hat{\Gamma}_{j t}=\sum_{i \in \mathcal{L}} r_{i 1}^{\hat{\alpha}} c_{i}+\sum_{t \in \mathcal{T}} \sum_{j \in \mathcal{J}} p_{j t} L_{j t}^{\hat{\alpha}}=$ $V_{1}^{\hat{\alpha}}(c) \geq \min _{\alpha \geq 0}\left\{V_{1}^{\alpha}(c)\right\}$.

Therefore, the optimal objective value of problem (6) can be obtained by solving problem (8)(12). The proof of Proposition 3 also implies that if $\left\{\hat{\rho}_{i t}: i \in \mathcal{L}, t \in \mathcal{T}\right\},\left\{\hat{\Gamma}_{j t}: j \in \mathcal{J}, t \in \mathcal{T}\right\}$, $\hat{\alpha}=\left\{\hat{\alpha}_{i j t}: i \in \mathcal{L}, j \in \mathcal{J}, t \in \mathcal{T}\right\}$ is an optimal solution to problem (8)-(12), then $\hat{\alpha}$ is an optimal solution to problem (6).

The fact that $V_{1}^{\alpha}(c)$ is linear in $c$ plays a crucial role in the tractability of the linear program in (8)-(12). In particular, it is important to note that the numbers of decision variables and constraints in problem (8)-(12) do not depend on the capacities of the flight legs. On the other hand, Adelman and Mersereau (2008) show that if $V_{1}^{\alpha}(c)$ were not linear in $c$, then the problem of finding the tightest possible upper bound could still be formulated as a linear program, but the numbers of decision variables and constraints in this linear program would increase linearly with the capacities. Problem (PL) in Adelman and Mersereau (2008) shows what this linear program would look like for a weakly coupled dynamic program over an infinite horizon and one can extend problem (PL) to the finite horizon case.

To gain some insight into the structure of problem (8)-(12), we associate the dual variables $\left\{y_{j t}\right.$ : $j \in \mathcal{J}, t \in \mathcal{T}\}$ with constraints (9)-(10) and the dual variables $\left\{z_{i t}: i \in \mathcal{L}, t \in \mathcal{T}\right\}$ with constraints (11). In this case, the dual of problem (8)-(12) can be written as

$$
\begin{array}{lll}
\hat{\zeta}=\max & \sum_{t \in \mathcal{T}} \sum_{j \in \mathcal{J}} f_{j} y_{j t} & \\
\text { subject to } & a_{i j} y_{j t}-p_{j t} z_{i 1}-\ldots-p_{j t} z_{i t} \leq 0 & \forall i \in \mathcal{L}, j \in \mathcal{J}, t \in \mathcal{T} \\
& z_{i 1}=c_{i} & \forall i \in \mathcal{L} \\
& \sum_{j \in \mathcal{J}} a_{i j} y_{j, t-1}+z_{i t}=0 & \forall i \in \mathcal{L}, t \in \mathcal{T} \backslash\{1\} \\
& y_{j t} \leq p_{j t} & \forall j \in \mathcal{J}, t \in \mathcal{T} \\
& y_{j t} \geq 0, z_{i t} \text { is free } & \forall i \in \mathcal{L}, j \in \mathcal{J}, t \in \mathcal{T} .
\end{array}
$$


Substituting for the decision variables $\left\{z_{i t}: i \in \mathcal{L}, t \in \mathcal{T}\right\}$ in the first set of constraints above by using the second and third sets of constraints, the problem above becomes

$$
\begin{aligned}
\hat{\zeta}=\max & \sum_{t \in \mathcal{T}} \sum_{j \in \mathcal{J}} f_{j} y_{j t} & \\
\text { subject to } & \sum_{k \in \mathcal{J}} p_{j t} a_{i k} y_{k 1}+\ldots+\sum_{k \in \mathcal{J}} p_{j t} a_{i k} y_{k, t-1}+a_{i j} y_{j t} \leq p_{j t} c_{i} & \forall i \in \mathcal{L}, j \in \mathcal{J}, t \in \mathcal{T} \\
& y_{j t} \leq p_{j t} & \forall j \in \mathcal{J}, t \in \mathcal{T} \\
& y_{j t} \geq 0 & \forall j \in \mathcal{J}, t \in \mathcal{T} .
\end{aligned}
$$

We have $V_{1}(c) \leq \min _{\alpha \geq 0}\left\{V_{1}^{\alpha}(c)\right\}$ by Proposition 2 and $\hat{\zeta}=\min _{\alpha \geq 0}\left\{V_{1}^{\alpha}(c)\right\}$ by Proposition 3. Therefore, we can obtain an upper bound on the optimal total expected revenue by solving problem (13)-(16).

There is an appealing interpretation for problem (13)-(16). The decision variable $y_{j t}$ in this problem corresponds to the probability that we accept a request for itinerary $j$ at time period $t$. In this case, the objective function accounts for the total expected revenue over the planning horizon. Constraints (15) ensure that the probability that we accept a request for itinerary $j$ at time period $t$ does not exceed the probability that a request for itinerary $j$ arrives at time period $t$. The interpretation of constraints (14) is a bit more intricate. If we write constraints (14) as

$$
a_{i j} \frac{y_{j t}}{p_{j t}} \leq c_{i}-\sum_{k \in \mathcal{J}} a_{i k} y_{k 1}-\ldots-\sum_{k \in \mathcal{J}} a_{i k} y_{k, t-1} \quad \forall i \in \mathcal{L}, j \in \mathcal{J}, t \in \mathcal{T},
$$

then the right side of constraints (17) is the expected remaining capacity on flight leg $i$ at time period $t$. The term $y_{j t} / p_{j t}$ on the left side of constraints (17) is the conditional probability that we accept a request for itinerary $j$ at time period $t$ given that there is a request for itinerary $j$ at time period $t$. Therefore, constraints (17) ensure that the expected capacity consumed on flight leg $i$, given that there is a request for itinerary $j$ at time period $t$, does not exceed the expected remaining capacity on flight leg $i$ at time period $t$.

Kunnumkal and Topaloglu (2008b) extend problem (13)-(16) to the customer choice setting. In this case, the number of constraints remains essentially the same, but the number of decision variables increases exponentially with the number of itineraries. They deal with the large number of decision variables by using column generation, but their column generation subproblem ends up being a nontrivial mixed integer linear program.

\section{Capacity-Dependent Bid Prices}

The method that we describe in Sections 2 and 3 computes dynamic bid prices. However, one intuitively expects that the opportunity cost of a unit of capacity on a flight leg should not only decrease as we approach the departure time, but it should also increase as the capacity on the flight leg becomes scarce. In other words, the bid prices should depend both on how much time is left until departure and on how many units of capacity are left on the flight legs. In this section, we develop a method that computes dynamic and capacity-dependent bid prices by decomposing the network revenue management problem 
into a number of single leg revenue management problems. This method is closely related to the popular dynamic programming decomposition idea; see Section 3.4.4 in Talluri and van Ryzin (2004).

We let $\hat{\alpha}=\left\{\hat{\alpha}_{i j t}: i \in \mathcal{L}, j \in \mathcal{J}, t \in \mathcal{T}\right\}$ be the optimal values of the dual variables associated with constraints (14) in problem (13)-(16). We choose a flight leg $i$ and letting $\overline{\mathcal{L}}_{i}=\mathcal{L} \backslash\{i\}$ for notational brevity, we relax constraints (14) for all other flight legs by associating the dual multipliers $\left\{\hat{\alpha}_{l j t}: l \in \overline{\mathcal{L}}_{i}, j \in \mathcal{J}, t \in \mathcal{T}\right\}$ with them. In this case, the objective function of problem (13)-(16) can be written as

$$
\sum_{t \in \mathcal{T}} \sum_{j \in \mathcal{J}} f_{j} y_{j t}+\sum_{t \in \mathcal{T}} \sum_{j \in \mathcal{J}} \sum_{l \in \overline{\mathcal{L}}_{i}}\left[p_{j t} c_{l}-\sum_{k \in \mathcal{J}} p_{j t} a_{l k} y_{k 1}-\ldots-\sum_{k \in \mathcal{J}} p_{j t} a_{l k} y_{k, t-1}-a_{l j} y_{j t}\right] \hat{\alpha}_{l j t} .
$$

Arranging the terms, it is easy to see that the expression above becomes

$$
\begin{aligned}
& \sum_{t \in \mathcal{T}} \sum_{j \in \mathcal{J}}\left[f_{j}-\sum_{l \in \overline{\mathcal{L}}_{i}} a_{l j} \hat{\alpha}_{l j t}\right] y_{j t}+\sum_{t \in \mathcal{T}} \sum_{j \in \mathcal{J}} \sum_{l \in \overline{\mathcal{L}}_{i}} p_{j t} c_{l} \hat{\alpha}_{l j t} \\
&-\sum_{t \in \mathcal{T}} \sum_{k \in \mathcal{J}} \sum_{l \in \overline{\mathcal{L}}_{i}} a_{l k} y_{k t}\left[\sum_{j \in \mathcal{J}} p_{j t} \hat{\alpha}_{l j, t+1}+\ldots+\sum_{j \in \mathcal{J}} p_{j t} \hat{\alpha}_{l j \tau}\right] .
\end{aligned}
$$

Noting the definition of $r_{i t}^{\alpha}$ and arranging the terms above once more, the objective function of problem (13)-(16) can finally be written as

$$
\sum_{t \in \mathcal{T}} \sum_{j \in \mathcal{J}}\left[f_{j}-\sum_{l \in \overline{\mathcal{L}}_{i}} a_{l j} \hat{\alpha}_{l j t}-\sum_{l \in \overline{\mathcal{L}}_{i}} a_{l j} r_{l, t+1}^{\hat{\alpha}}\right] y_{j t}+\sum_{l \in \overline{\mathcal{L}}_{i}} r_{l 1}^{\hat{\alpha}} c_{l}
$$

Therefore, the duality theory implies that the linear program

$$
\begin{aligned}
& \hat{\zeta}=\max \sum_{t \in \mathcal{T}} \sum_{j \in \mathcal{J}}\left[f_{j}-\sum_{l \in \overline{\mathcal{L}}_{i}} a_{l j} \hat{\alpha}_{l j t}-\sum_{l \in \overline{\mathcal{L}}_{i}} a_{l j} r_{l, t+1}^{\hat{\alpha}}\right] y_{j t}+\sum_{l \in \overline{\mathcal{L}}_{i}} r_{l 1}^{\hat{\alpha}} c_{l} \\
& \text { subject to } \sum_{k \in \mathcal{J}} p_{j t} a_{i k} y_{k 1}+\ldots+\sum_{k \in \mathcal{J}} p_{j t} a_{i k} y_{k, t-1}+a_{i j} y_{j t} \leq p_{j t} c_{i} \quad \forall j \in \mathcal{J}, t \in \mathcal{T} \\
& y_{j t} \leq p_{j t} \\
& \forall j \in \mathcal{J}, t \in \mathcal{T} \\
& y_{j t} \geq 0 \\
& \forall j \in \mathcal{J}, t \in \mathcal{T}
\end{aligned}
$$

has the same optimal objective value as problem (13)-(16).

Ignoring the constant term $\sum_{l \in \overline{\mathcal{L}}_{i}} r_{l 1}^{\hat{\alpha}} c_{l}$ in the objective function, problem (18)-(21) has the same structure as problem (13)-(16), but problem (18)-(21) focuses on a single leg revenue management problem that takes place over flight leg $i$ under the assumption that

$$
f_{j}-\sum_{l \in \overline{\mathcal{L}}_{i}} a_{l j} \hat{\alpha}_{l j t}-\sum_{l \in \overline{\mathcal{L}}_{i}} a_{l j} r_{l, t+1}^{\hat{\alpha}}
$$

is the revenue associated with itinerary $j$ at time period $t$. Therefore, $\hat{\zeta}-\sum_{l \in \overline{\mathcal{L}}_{i}} r_{l 1}^{\hat{\alpha}} c_{l}$ is an upper bound on the optimal total expected revenue for the single leg revenue management problem that takes place over flight leg $i$. On the other hand, we can obtain the optimal total expected revenue for the single leg revenue management problem that takes place over flight leg $i$ by solving the optimality equation

$$
v_{i t}\left(x_{i t}\right)=\max _{u_{t} \in \mathcal{U}_{i}\left(x_{i t}\right)}\left\{\sum_{j \in \mathcal{J}} p_{j t}\left\{\left[f_{j}-\sum_{l \in \overline{\mathcal{L}}_{i}} a_{l j} \hat{\alpha}_{l j t}-\sum_{l \in \overline{\mathcal{L}}_{i}} a_{l j} r_{l, t+1}^{\hat{\alpha}}\right] u_{j t}+v_{i, t+1}\left(x_{i t}-a_{i j} u_{j t}\right)\right\}\right\},
$$


where we let $\mathcal{U}_{i}\left(x_{i t}\right)=\left\{u_{t} \in\{0,1\}^{|\mathcal{J}|}: a_{i j} u_{j t} \leq x_{i t} \forall j \in \mathcal{J}\right\}$ and use an optimality equation that is similar to the one in (1), but focus only on flight leg $i$.

We have $v_{i 1}\left(c_{i}\right) \leq \hat{\zeta}-\sum_{l \in \overline{\mathcal{L}}_{i}} r_{l 1}^{\hat{\alpha}} c_{l}$ by the discussion in the paragraph above. Furthermore, the next proposition shows that $V_{1}(c) \leq v_{i 1}\left(c_{i}\right)+\sum_{l \in \overline{\mathcal{L}}_{i}} r_{l 1}^{\hat{\alpha}} c_{l}$. Therefore, we have

$$
V_{1}(c) \leq v_{i 1}\left(c_{i}\right)+\sum_{l \in \overline{\mathcal{L}}_{i}} r_{l 1}^{\hat{\alpha}} c_{l} \leq \hat{\zeta}
$$

so that $v_{i 1}\left(c_{i}\right)+\sum_{l \in \overline{\mathcal{L}}_{i}} r_{l 1}^{\hat{\alpha}} c_{l}$ is an upper bound on the optimal total expected revenue and this upper bound is tighter than the one provided by the optimal objective value of problem (13)-(16).

Proposition 4 If $\hat{\alpha}=\left\{\hat{\alpha}_{i j t}: i \in \mathcal{L}, j \in \mathcal{J}, t \in \mathcal{T}\right\}$ are the optimal values of the dual variables associated with constraints (14) in problem (13)-(16), then we have $V_{t}\left(x_{t}\right) \leq v_{i t}\left(x_{i t}\right)+\sum_{l \in \overline{\mathcal{L}}_{i}} r_{l t}^{\hat{\alpha}} x_{l t}$ for all $t \in \mathcal{T}$.

Proof We show the result by induction over the time periods. It is easy to show the result for the last time period. Assuming that the result holds for time period $t+1$ and letting $\hat{u}_{t}=\left\{\hat{u}_{j t}: j \in \mathcal{J}\right\}$ be an optimal solution to problem (1), we have

$$
\begin{aligned}
V_{t}\left(x_{t}\right) & =\sum_{j \in \mathcal{J}} p_{j t}\left[f_{j} \hat{u}_{j t}+V_{t+1}\left(x_{t}-\hat{u}_{j t} \sum_{i \in \mathcal{L}} a_{i j} e_{i}\right)\right] \\
& \leq \sum_{j \in \mathcal{J}} p_{j t}\left\{\left[f_{j}-\sum_{l \in \overline{\mathcal{L}}_{i}} a_{l j} \hat{\alpha}_{l j t}\right] \hat{u}_{j t}+\sum_{l \in \overline{\mathcal{L}}_{i}} \hat{\alpha}_{l j t} x_{l t}+v_{i, t+1}\left(x_{i t}-a_{i j} \hat{u}_{j t}\right)+\sum_{l \in \overline{\mathcal{L}}_{i}} r_{l, t+1}^{\hat{\alpha}}\left[x_{l t}-a_{l j} \hat{u}_{j t}\right]\right\} \\
& =\sum_{j \in \mathcal{J}} p_{j t}\left\{\left[f_{j}-\sum_{l \in \overline{\mathcal{L}}_{i}} a_{l j} \hat{\alpha}_{l j t}-\sum_{l \in \overline{\mathcal{L}}_{i}} a_{l j} r_{l, t+1}^{\hat{\alpha}}\right] \hat{u}_{j t}+v_{i, t+1}\left(x_{i t}-a_{i j} \hat{u}_{j t}\right)\right\}+\sum_{l \in \overline{\mathcal{L}}_{i}} r_{l t}^{\hat{\alpha}} x_{l t}, \\
& \leq v_{i t}\left(x_{i t}\right)+\sum_{l \in \overline{\mathcal{L}}_{i}} r_{l t}^{\hat{\alpha}} x_{l t},
\end{aligned}
$$

where the first inequality follows from the induction assumption and the fact that $\hat{\alpha}_{l j t} \geq 0$ and $a_{l j} \hat{u}_{j t} \leq$ $x_{l t}$ for all $l \in \overline{\mathcal{L}}_{i}, j \in \mathcal{J}$, the second equality follows from the fact that $r_{i t}^{\alpha}=\sum_{j \in \mathcal{J}} p_{j t} \alpha_{i j t}+r_{i, t+1}^{\alpha}$ and the second inequality follows from the fact that $\hat{u}_{t}$ is a feasible but not necessarily an optimal solution to problem (22).

Dynamic programming decomposition ideas date back to Belobaba (1987), but the fact that these ideas can provide upper bounds on the optimal total expected revenue is recently shown by Zhang and Adelman (2006). Proposition 4 shows that a dynamic programming decomposition idea in conjunction with the dynamic bid prices computed by our method can provide upper bounds on the optimal total expected revenue. Our induction proof mimics the one in Zhang and Adelman (2006), but the relaxation argument that we use on problem (13)-(16) is new and it clearly demonstrates why we need to associate the revenue $f_{j}-\sum_{l \in \overline{\mathcal{L}}_{i}} a_{l j} \hat{\alpha}_{l j t}-\sum_{l \in \overline{\mathcal{L}}_{i}} a_{l j} r_{l, t+1}^{\hat{\alpha}}$ with itinerary $j$ at time period $t$. The term $f_{j}-$ $\sum_{l \in \overline{\mathcal{L}}_{i}} a_{l j} \hat{\alpha}_{l j t}-\sum_{l \in \overline{\mathcal{L}}_{i}} a_{l j} r_{l, t+1}^{\hat{\alpha}}$ is quite nonintuitive and it is not easy to come up with this term without following our relaxation argument. 
Using Proposition 4 for all of the flight legs, the tightest possible upper bound on the optimal total expected revenue is $\min _{i \in \mathcal{L}}\left\{v_{i 1}\left(c_{i}\right)+\sum_{l \in \overline{\mathcal{L}}_{i}} r_{l 1}^{\hat{\alpha}} c_{l}\right\}$. Furthermore, we can collect the one-dimensional value functions $\left\{v_{i t}(\cdot): i \in \mathcal{L}, t \in \mathcal{T}\right\}$ together to construct the separable value function approximation $\tilde{V}_{t}\left(x_{t}\right)=\sum_{i \in \mathcal{L}} v_{i t}\left(x_{i t}\right)$ for all $t \in \mathcal{T}$. In this case, we make the itinerary acceptance decisions by replacing $\left\{V_{t}(\cdot): t \in \mathcal{T}\right\}$ on the right side of $(2)$ with $\left\{\sum_{i \in \mathcal{L}} v_{i t}(\cdot): t \in \mathcal{T}\right\}$. Therefore, if we have

$$
f_{j}+\sum_{i \in \mathcal{L}} v_{i, t+1}\left(x_{i t}-a_{i j}\right) \geq \sum_{i \in \mathcal{L}} v_{i, t+1}\left(x_{i t}\right)
$$

and $a_{i j} \leq x_{i t}$ for all $i \in \mathcal{L}$, then we accept a request for itinerary $j$ at time period $t$. This is essentially the same approximation used by the dynamic programming decomposition idea. Noting that $v_{i, t+1}\left(x_{i t}\right)-$ $v_{i, t+1}\left(x_{i t}-a_{i j}\right)=\sum_{q=1}^{a_{i j}}\left[v_{i, t+1}\left(x_{i t}+1-q\right)-v_{i, t+1}\left(x_{i t}-q\right)\right]$, the decision rule above is equivalent to accepting a request for itinerary $j$ at time period $t$ when we have

$$
f_{j} \geq \sum_{i \in \mathcal{L}} \sum_{q=1}^{a_{i j}}\left[v_{i, t+1}\left(x_{i t}+1-q\right)-v_{i, t+1}\left(x_{i t}-q\right)\right]
$$

and $a_{i j} \leq x_{i t}$ for all $i \in \mathcal{L}$.

We can view $v_{i, t+1}\left(x_{i t}\right)-v_{i, t+1}\left(x_{i t}-1\right)$ as the bid price associated with the $x_{i t}$-th unit of capacity on flight leg $i$ at time period $t$. Comparing the decision rule in (24) with the one in (5), we note that the bid prices in (5) are dynamic, whereas the bid prices in (24) are both dynamic and capacity-dependent. It is also possible to show that $\left\{v_{i t}(\cdot): i \in \mathcal{L}, t \in \mathcal{T}\right\}$ are concave functions and this implies that the bid price associated with a flight leg increases as the capacity on the flight leg becomes scarce. This is in agreement with the intuitive expectation that we mention at the beginning of this section.

\section{Other Approaches for Computing Bid Prices}

There exist a number of other approaches in the network revenue management literature that can be used to compute bid prices. In this section, we review some of these approaches and compare them with the two methods that we develop in Sections 2-4. All of the approaches that we review in this section are used as benchmark methods in our computational experiments.

\subsection{Deterministic Linear Program}

A traditional approach for computing bid prices involves solving a deterministic linear program. Letting $w_{j}$ be the number of requests for itinerary $j$ that we plan to accept over the planning horizon, this linear program has the form

$$
\begin{array}{rlr}
\max & \sum_{j \in \mathcal{J}} f_{j} w_{j} & \\
\text { subject to } & \sum_{j \in \mathcal{J}} a_{i j} w_{j} \leq c_{i} & \forall i \in \mathcal{L} \\
& w_{j} \leq \sum_{t \in \mathcal{T}} p_{j t} & \forall j \in \mathcal{J} \\
& w_{j} \geq 0 & \forall j \in \mathcal{J} .
\end{array}
$$


Constraints (26) ensure that the numbers of itinerary requests that we plan to accept do not violate the leg capacities, whereas constraints (27) ensure that we do not plan to accept more itinerary requests than the expected numbers of itinerary requests. The linear program above dates back to Simpson (1989) and Williamson (1992).

Letting $\left\{\hat{\mu}_{i}: i \in \mathcal{L}\right\}$ be the optimal values of the dual variables associated with constraints (26) in problem (25)-(28), we can use $\hat{\mu}_{i}$ as an estimate of the opportunity cost of a unit of capacity on flight leg $i$. In other words, we can use $\hat{\mu}_{i}$ as the bid price associated with flight leg $i$. In this case, we accept a request for itinerary $j$ at time period $t$ when we have

$$
f_{j} \geq \sum_{i \in \mathcal{L}} a_{i j} \hat{\mu}_{i}
$$

and $a_{i j} \leq x_{i t}$ for all $i \in \mathcal{L}$. Letting $\tilde{V}_{t}\left(x_{t}\right)=\sum_{i \in \mathcal{L}} \hat{\mu}_{i} x_{i t}$ for all $t \in \mathcal{T}$, since $\tilde{V}_{t+1}\left(x_{t}\right)-\tilde{V}_{t+1}\left(x_{t}-\right.$ $\left.\sum_{i \in \mathcal{L}} a_{i j} e_{i}\right)=\sum_{i \in \mathcal{L}} a_{i j} \hat{\mu}_{i}$, it is easy to see that the decision rule in (29) is equivalent to approximating $\left\{V_{t}(\cdot): t \in \mathcal{T}\right\}$ in $(2)$ by $\left\{\tilde{V}_{t}(\cdot): t \in \mathcal{T}\right\}$. We emphasize that the bid prices in (29) do not depend on how much time is left until departure or on how many units of capacity are left on the flight legs.

It is possible to show that the optimal objective value of problem (25)-(28) provides an upper bound on the optimal total expected revenue; see Bertsimas and Popescu (2003). In other words, letting $\hat{Z}$ be the optimal objective value of problem (25)-(28), we have $V_{1}(c) \leq \hat{Z}$. The next proposition shows that $V_{1}(c) \leq \hat{\zeta} \leq \hat{Z}$. Therefore, the upper bound on the optimal total expected revenue provided by problem (13)-(16) is tighter than the one provided by problem (25)-(28).

Proposition 5 We have $V_{1}(c) \leq \hat{\zeta} \leq \hat{Z}$.

Proof The proof follows from an aggregation argument similar to the proof of Theorem 1 in Adelman (2007). Propositions 2 and 3 show that $V_{1}(c) \leq \hat{\zeta}$ and we focus only on the second inequality. We let $\hat{y}=\left\{\hat{y}_{j t}: j \in \mathcal{J}, t \in \mathcal{T}\right\}$ be an optimal solution to problem (13)-(16) and define the solution $\hat{w}=\left\{\hat{w}_{j}: j \in \mathcal{J}\right\}$ as $\hat{w}_{j}=\sum_{t \in \mathcal{T}} \hat{y}_{j t}$ for all $j \in \mathcal{J}$. Since $\hat{y}$ is a feasible solution to problem (13)-(16) and $\sum_{j \in \mathcal{J}} p_{j \tau}=1$, adding constraints (14) for flight leg $i$ and time period $\tau$ over all $j \in \mathcal{J}$, we have

$$
c_{i}=\sum_{j \in \mathcal{J}} p_{j \tau} c_{i} \geq \sum_{j \in \mathcal{J}} p_{j \tau}\left[\sum_{k \in \mathcal{J}} a_{i k} \hat{y}_{k 1}+\ldots+\sum_{k \in \mathcal{J}} a_{i k} \hat{y}_{k, \tau-1}\right]+\sum_{j \in \mathcal{J}} a_{i j} \hat{y}_{j \tau}=\sum_{t \in \mathcal{T}} \sum_{j \in \mathcal{J}} a_{i j} \hat{y}_{j t}
$$

for all $i \in \mathcal{L}$. Similarly, adding constraints (15) for itinerary $j$ over all $t \in \mathcal{T}$, we have $\sum_{t \in \mathcal{T}} \hat{y}_{j t} \leq$ $\sum_{t \in \mathcal{T}} p_{j t}$ for all $j \in \mathcal{J}$. Therefore, $\hat{w}$ is a feasible solution to problem $(25)-(28)$ and we have $\hat{\zeta}=$ $\sum_{t \in \mathcal{T}} \sum_{j \in \mathcal{J}} f_{j} \hat{y}_{j t}=\sum_{j \in \mathcal{J}} f_{j} \hat{w}_{j} \leq \hat{Z}$.

Comparing problem (25)-(28) with problem (13)-(16), we note that the main difference between the two problems is in the way in which they capture the capacity availabilities. Problem (13)-(16) has one capacity constraint for each flight leg, for each itinerary and for each time period, whereas problem (25)-(28) has one capacity constraint for each flight leg. Therefore, the capacity constraints in problem (13)-(16) operate at a more disaggregate level than those in problem (25)-(28) and the upper bound provided by problem (13)-(16) ends up being tighter. 


\subsection{Dynamic Programming Decomposition}

This method is similar to the one in Section 4 and it computes dynamic and capacity-dependent bid prices by decomposing the network revenue management problem into a number of single leg revenue management problems. In particular, letting $\left\{\hat{\mu}_{i}: i \in \mathcal{L}\right\}$ be the optimal values of the dual variables associated with constraints (26) in problem (25)-(28), we consider the single leg revenue management problem that takes place over flight leg $i$ under the assumption that $f_{j}-\sum_{l \in \overline{\mathcal{L}}_{i}} a_{l j} \hat{\mu}_{l}$ is the revenue associated with itinerary $j$. The optimal total expected revenue for the single leg revenue management problem that takes place over flight leg $i$ can be obtained by solving the optimality equation

$$
\vartheta_{i t}\left(x_{i t}\right)=\max _{u_{t} \in \mathcal{U}_{i}\left(x_{i t}\right)}\left\{\sum_{j \in \mathcal{J}} p_{j t}\left\{\left[f_{j}-\sum_{l \in \overline{\mathcal{L}}_{i}} a_{l j} \hat{\mu}_{l}\right] u_{j t}+\vartheta_{i, t+1}\left(x_{i t}-a_{i j} u_{j t}\right)\right\}\right\} .
$$

Using a relaxation argument that is similar to the one in Section 4, but working with problem (25)-(28) and dual multipliers $\left\{\hat{\mu}_{l}: l \in \overline{\mathcal{L}}_{i}\right\}$ instead of problem (13)-(16) and dual multipliers $\left\{\hat{\alpha}_{l j t}: l \in \overline{\mathcal{L}}_{i}, j \in\right.$ $\mathcal{J}, t \in \mathcal{T}\}$, it is possible to show that

$$
V_{1}(c) \leq \vartheta_{i 1}\left(c_{i}\right)+\sum_{l \in \overline{\mathcal{L}}_{i}} \hat{\mu}_{l} c_{l} \leq \hat{Z}
$$

Therefore, we can solve the optimality equation in (30) to obtain an upper bound on the optimal total expected revenue that is tighter than the one provided by problem (25)-(28). We note that the chain of inequalities in (31) is analogous to the one in (23). This result was first shown by Zhang and Adelman (2006), but without using the relaxation argument that we use in this paper.

Repeating this approach for all of the flight legs, the tightest possible upper bound on the optimal total expected revenue is $\min _{i \in \mathcal{L}}\left\{\vartheta_{i 1}\left(c_{i}\right)+\sum_{l \in \overline{\mathcal{L}}_{i}} \hat{\mu}_{l} c_{l}\right\}$. Furthermore, we can collect the one-dimensional value functions $\left\{\vartheta_{i t}(\cdot): i \in \mathcal{L}, t \in \mathcal{T}\right\}$ together to construct the separable value function approximation

$\tilde{V}_{t}\left(x_{t}\right)=\sum_{i \in \mathcal{L}} \vartheta_{i t}\left(x_{i t}\right)$ for all $t \in \mathcal{T}$; see Section 3.4.4 in Talluri and van Ryzin (2004). In this case, replacing $\left\{V_{t}(\cdot): t \in \mathcal{T}\right\}$ on the right side of $(2)$ with $\left\{\sum_{i \in \mathcal{L}} \vartheta_{i t}(\cdot): t \in \mathcal{T}\right\}$, if we have

$$
f_{j}+\sum_{i \in \mathcal{L}} \vartheta_{i, t+1}\left(x_{i t}-a_{i j}\right) \geq \sum_{i \in \mathcal{L}} \vartheta_{i, t+1}\left(x_{i t}\right)
$$

and $a_{i j} \leq x_{i t}$ for all $i \in \mathcal{L}$, then we accept a request for itinerary $j$ at time period $t$. We note that the decision rule above is equivalent to using dynamic and capacity-dependent bid prices.

\subsection{Decomposition by Revenue Allocation}

This approach was developed by Topaloglu (2009) to compute dynamic and capacity-dependent bid prices. Kunnumkal and Topaloglu (2007) show that it is possible to extend this approach to the customer choice setting. The main idea behind this approach is to allocate the revenue associated with an itinerary among the different flight legs. In particular, we let $\beta_{i j t}$ be the portion of the revenue generated from accepting a request for itinerary $j$ at time period $t$ that is allocated to flight leg $i$. We do not specify yet how the revenue allocations are chosen, but they satisfy

$$
\sum_{i \in \mathcal{L}} \beta_{i j t}=f_{j} \quad \forall j \in \mathcal{J}, t \in \mathcal{T} .
$$


Allocating the revenues in this fashion immediately allows us to formulate single leg revenue management problems. In the single leg revenue management problem that takes place over flight leg $i$, the revenue associated with itinerary $j$ at time period $t$ is $\beta_{i j t}$. Therefore, we can solve the optimality equation

$$
\nu_{i t}^{\beta}\left(x_{i t}\right)=\max _{u_{t} \in \mathcal{U}_{i}\left(x_{i t}\right)}\left\{\sum_{j \in \mathcal{J}} p_{j t}\left[\beta_{i j t} u_{j t}+\nu_{i, t+1}^{\beta}\left(x_{i t}-a_{i j} u_{j t}\right)\right]\right\}
$$

to obtain the optimal total expected revenue for the single leg revenue management that takes place over flight leg $i$. We use the superscript $\beta=\left\{\beta_{i j t}: i \in \mathcal{L}, j \in \mathcal{J}, t \in \mathcal{T}\right\}$ in the value functions to emphasize that the solution to the optimality equation above depends on the revenue allocations.

The next proposition shows that we can obtain upper bounds on the value functions by solving the optimality equation in (33). Proposition 1 in Kunnumkal and Topaloglu (2007) shows this result in the customer choice setting. Since the proof of this result is crucial for demonstrating why the revenue allocations need to satisfy (32), we simplify the proof in Kunnumkal and Topaloglu (2007) to capture the independent demand setting here.

Proposition 6 If $\beta$ satisfies (32), then we have $V_{t}\left(x_{t}\right) \leq \sum_{i \in \mathcal{L}} \nu_{i t}^{\beta}\left(x_{i t}\right)$ for all $t \in \mathcal{T}$.

Proof We show the result by induction over the time periods. It is easy to show the result for the last time period. Assuming that the result holds for time period $t+1$ and letting $\hat{u}_{t}=\left\{\hat{u}_{j t}: j \in \mathcal{J}\right\}$ be an optimal solution to problem (1), we have

$$
\begin{aligned}
V_{t}\left(x_{t}\right)=\sum_{j \in \mathcal{J}} p_{j t}\left[\sum_{i \in \mathcal{L}} \beta_{i j t} \hat{u}_{j t}+\right. & \left.V_{t+1}\left(x_{t}-\hat{u}_{j t} \sum_{i \in \mathcal{L}} a_{i j} e_{i}\right)\right] \\
& \leq \sum_{j \in \mathcal{J}} p_{j t}\left[\sum_{i \in \mathcal{L}} \beta_{i j t} \hat{u}_{j t}+\sum_{i \in \mathcal{L}} \nu_{i, t+1}^{\beta}\left(x_{i t}-a_{i j} \hat{u}_{j t}\right)\right] \leq \sum_{i \in \mathcal{L}} \nu_{i t}^{\beta}\left(x_{i t}\right),
\end{aligned}
$$

where the first equality follows from (32), the first inequality follows from the induction assumption and the second inequality follows from the fact that $\hat{u}_{t}$ is a feasible but not necessarily an optimal solution to problem (33).

Therefore, $V_{1}(c)$ is bounded from above by $\sum_{i \in \mathcal{L}} \nu_{i 1}^{\beta}\left(c_{i}\right)$ as long as the revenue allocations satisfy (32). To obtain the tightest possible upper bound on $V_{1}(c)$, we can solve the problem

$$
\min _{\beta \in \mathcal{F}}\left\{\sum_{i \in \mathcal{L}} \nu_{i 1}^{\beta}\left(c_{i}\right)\right\}
$$

where $\mathcal{F}=\left\{\beta: \sum_{i \in \mathcal{L}} \beta_{i j t}=f_{j} \forall j \in \mathcal{J}, t \in \mathcal{T}\right\}$. Proposition 3 in Topaloglu (2009) shows that $\nu_{i 1}^{\beta}\left(c_{i}\right)$ is a convex function of $\beta$ and we can solve problem (34) by using subgradient optimization or Benders decomposition; see Wolsey (1998) and Ruszczynski (2003).

We note that problem (34) is analogous to problem (6). However, there does not exist a closed form expression for $\sum_{i \in \mathcal{L}} \nu_{i 1}^{\beta}\left(c_{i}\right)$ comparable to (4) and solving problem (34) is computationally much more expensive than solving problem (6). The next proposition shows that the extra computational burden pays off and the upper bound on the optimal total expected revenue provided by problem (34) is tighter than the one provided by problem (6). 
Proposition 7 If $\hat{\alpha}=\left\{\hat{\alpha}_{i j t}: i \in \mathcal{L}, j \in \mathcal{J}, t \in \mathcal{T}\right\}$ are the optimal values of the dual variables associated with constraints (14) in problem (13)-(16), then we have

$$
\min _{\beta \in \mathcal{F}}\left\{\sum_{i \in \mathcal{L}} \nu_{i 1}^{\beta}\left(c_{i}\right)\right\} \leq \min _{i \in \mathcal{L}}\left\{v_{i 1}\left(c_{i}\right)+\sum_{l \in \overline{\mathcal{L}}_{i}} r_{l 1}^{\hat{\alpha}} c_{l}\right\} \leq \min _{\alpha \geq 0}\left\{V_{1}^{\alpha}(c)\right\} .
$$

Proof Proposition 3 and the second inequality in (23) show that the second inequality above holds and we focus only on the first inequality here. We let $\hat{\alpha}$ be an optimal solution to problem (6). We choose a flight leg $i$ and let $\hat{\beta}_{i j t}=f_{j}-\sum_{l \in \overline{\mathcal{L}}_{i}} a_{l j} \hat{\alpha}_{l j t}-\sum_{l \in \overline{\mathcal{L}}_{i}} a_{l j} r_{l, t+1}^{\hat{\alpha}}$ for all $j \in \mathcal{J}, t \in \mathcal{T}$ and $\hat{\beta}_{l j t}=a_{l j} \hat{\alpha}_{l j t}+a_{l j} r_{l, t+1}^{\hat{\alpha}}$ for all $l \in \overline{\mathcal{L}}_{i}, j \in \mathcal{J}, t \in \mathcal{T}$. We note that $\hat{\beta}=\left\{\hat{\beta}_{i j t}: i \in \mathcal{L}, j \in \mathcal{J}, t \in \mathcal{T}\right\} \in \mathcal{F}$. The proof shows that $\sum_{l \in \mathcal{L}} \nu_{l t}^{\hat{\beta}}\left(x_{l t}\right) \leq v_{i t}\left(x_{i t}\right)+\sum_{l \in \overline{\mathcal{L}}_{i}} r_{l t}^{\hat{\alpha}} x_{l t}$ for all $t \in \mathcal{T}$. In this case, the result follows from the fact that flight leg $i$ is arbitrary and $\hat{\beta} \in \mathcal{F}$.

First, if we let $\beta_{i j t}=\hat{\beta}_{i j t}$ for all $j \in \mathcal{J}, t \in \mathcal{T}$ in (33), then the optimality equations in (22) and (33) become identical. Therefore, we have $\nu_{i t}^{\hat{\beta}}\left(x_{i t}\right)=v_{i t}\left(x_{i t}\right)$ for all $t \in \mathcal{T}$. Second, we show by induction over the time periods that $\nu_{l t}^{\hat{\beta}}\left(x_{l t}\right) \leq r_{l t}^{\hat{\alpha}} x_{l t}$ for all $l \in \overline{\mathcal{L}}_{i}, t \in \mathcal{T}$. It is easy to show the result for the last time period. Assuming that the result holds for time period $t+1$, we let $\hat{u}_{t}=\left\{\hat{u}_{j t}: j \in \mathcal{J}\right\}$ be an optimal solution to problem (33) when we solve this problem for flight leg $l$ with $\beta=\hat{\beta}$. We have

$$
\begin{array}{r}
\nu_{l t}^{\hat{\beta}}\left(x_{l t}\right)=\sum_{j \in \mathcal{J}} p_{j t}\left[\left[a_{l j} \hat{\alpha}_{l j t}+a_{l j} r_{l, t+1}^{\hat{\alpha}}\right] \hat{u}_{j t}+\nu_{l, t+1}^{\hat{\beta}}\left(x_{l t}-a_{l j} \hat{u}_{j t}\right)\right] \\
\leq \sum_{j \in \mathcal{J}} p_{j t}\left[\left[a_{l j} \hat{\alpha}_{l j t}+a_{l j} r_{l, t+1}^{\hat{\alpha}}\right] \hat{u}_{j t}+r_{l, t+1}^{\hat{\alpha}}\left[x_{l t}-a_{l j} \hat{u}_{j t}\right]\right] \\
\leq \sum_{j \in \mathcal{J}} p_{j t} \hat{\alpha}_{l j t} x_{l t}+r_{l, t+1}^{\hat{\alpha}} x_{l t}=r_{l t}^{\hat{\alpha}} x_{l t},
\end{array}
$$

where the first inequality follows from the induction assumption, the second inequality follows from the fact that $\hat{\alpha}_{l j t} \geq 0$ and $a_{l j} \hat{u}_{j t} \leq x_{l t}$ for all $j \in \mathcal{J}$ and the second equality follows from the fact that $r_{i t}^{\alpha}=\sum_{j \in \mathcal{J}} p_{j t} \alpha_{i j t}+r_{i, t+1}^{\alpha}$.

Kunnumkal and Topaloglu (2007) use the argument above to show a similar result in the customer choice setting. If their result were translated to the independent demand setting, then it would read as $\min _{\beta \in \mathcal{F}}\left\{\sum_{i \in \mathcal{L}} \nu_{i 1}^{\beta}\left(c_{i}\right)\right\} \leq \min _{i \in \mathcal{L}}\left\{\vartheta_{i 1}\left(c_{i}\right)+\sum_{l \in \overline{\mathcal{L}}_{i}} \hat{\mu}_{l} c_{l}\right\} \leq \hat{Z}$, where $\left\{\vartheta_{i t}(\cdot): i \in \mathcal{L}, t \in \mathcal{T}\right\}$ are computed through the optimality equation in $(30),\left\{\hat{\mu}_{i}: i \in \mathcal{L}\right\}$ are the optimal values of the dual variables associated with constraints (26) in problem (25)-(28) and $\hat{Z}$ is the optimal objective value of problem (25)-(28). Therefore, we can interpret Proposition 7 as the analogue of their result for the case where we compute dynamic bid prices through problem (13)-(16).

Letting $\hat{\beta}$ be an optimal solution to problem (34), we make the itinerary acceptance decisions by replacing $\left\{V_{t}(\cdot): t \in \mathcal{T}\right\}$ on the right side of $(2)$ with $\left\{\sum_{i \in \mathcal{L}} \nu_{i t}^{\hat{\beta}}(\cdot): t \in \mathcal{T}\right\}$. Therefore, if we have

$$
f_{j}+\sum_{i \in \mathcal{L}} \nu_{i, t+1}^{\hat{\beta}}\left(x_{i t}-a_{i j}\right) \geq \sum_{i \in \mathcal{L}} \nu_{i, t+1}^{\hat{\beta}}\left(x_{i t}\right)
$$

and $a_{i j} \leq x_{i t}$ for all $i \in \mathcal{L}$, then we accept a request for itinerary $j$ at time period $t$. Similar to the decision rules in Sections 4 and 5.2, the decision rule above is equivalent to using dynamic and capacity-dependent bid prices. 


\subsection{Linear Value Function Approximations}

Similar to the method that we describe in Sections 2 and 3, this method is directed at computing dynamic bid prices and it was proposed by Adelman (2007). Zhang and Adelman (2006) extend this method to the customer choice setting. We begin by letting $C=\max _{i \in \mathcal{L}}\left\{c_{i}\right\}$ and $\mathcal{C}=\{0, \ldots, C\}$ so that the remaining capacity on each flight leg is always in the $\operatorname{set} \mathcal{C}$ and we can use $\mathcal{C}^{|\mathcal{L}|}$ as the state space in the optimality equation in (1). In this case, Adelman (2007) shows that $V_{1}(c)$ can be computed by solving the linear program

$$
\min V_{1}(c)
$$

$$
\begin{array}{lll}
\text { subject to } & V_{t}\left(x_{t}\right) \geq \sum_{j \in \mathcal{J}} p_{j t}\left[f_{j} u_{j t}+V_{t+1}\left(x_{t}-u_{j t} \sum_{i \in \mathcal{L}} a_{i j} e_{i}\right)\right] & \forall x_{t} \in \mathcal{C}^{|\mathcal{L}|}, u_{t} \in \mathcal{U}\left(x_{t}\right), t \in \mathcal{T} \backslash\{\tau\} \\
V_{\tau}\left(x_{\tau}\right) \geq \sum_{j \in \mathcal{J}} p_{j \tau} f_{j} u_{j \tau} & \forall x_{\tau} \in \mathcal{C}^{|\mathcal{L}|}, u_{\tau} \in \mathcal{U}\left(x_{\tau}\right),
\end{array}
$$

where $\left\{V_{t}\left(x_{t}\right): x_{t} \in \mathcal{C}^{|\mathcal{L}|}, t \in \mathcal{T}\right\}$ are the decision variables. One approach to deal with the large number of decision variables in the problem above is to approximate the value functions by linear functions of the form $\hat{V}_{t}\left(x_{t}\right)=\theta_{t}+\sum_{i \in \mathcal{L}} \gamma_{i t} x_{i t}$. To decide what values to choose for $\left\{\theta_{t}: t \in \mathcal{T}\right\}$ and $\left\{\gamma_{i t}: i \in \mathcal{L}, t \in \mathcal{T}\right\}$, we replace $V_{t}\left(x_{t}\right)$ in the problem above with $\theta_{t}+\sum_{i \in \mathcal{L}} \gamma_{i t} x_{i t}$ to obtain the linear program

$$
\begin{aligned}
& \min \theta_{1}+\sum_{i \in \mathcal{L}} \gamma_{i 1} c_{i} \\
& \text { subject to } \theta_{t}+\sum_{i \in \mathcal{L}} \gamma_{i t} x_{i t} \geq \sum_{j \in \mathcal{J}} p_{j t}\left[f_{j} u_{j t}+\theta_{t+1}+\sum_{i \in \mathcal{L}} \gamma_{i, t+1}\left[x_{i t}-a_{i j} u_{j t}\right]\right] \\
& \forall x_{t} \in \mathcal{C}^{|\mathcal{L}|}, u_{t} \in \mathcal{U}\left(x_{t}\right), t \in \mathcal{T} \backslash\{\tau\} \\
& \theta_{\tau}+\sum_{i \in \mathcal{L}} \gamma_{i \tau} x_{i \tau} \geq \sum_{j \in \mathcal{J}} p_{j \tau} f_{j} u_{j \tau} \quad \forall x_{\tau} \in \mathcal{C}^{|\mathcal{L}|}, u_{\tau} \in \mathcal{U}\left(x_{\tau}\right),
\end{aligned}
$$

where $\left\{\theta_{t}: t \in \mathcal{T}\right\}$ and $\left\{\gamma_{i t}: i \in \mathcal{L}, t \in \mathcal{T}\right\}$ are the decision variables. The number of decision variables in problem (36)-(38) is manageable and we can deal with the large number of constraints by using column generation on the dual.

Letting $\left\{\hat{\theta}_{t}: t \in \mathcal{T}\right\}$ and $\left\{\hat{\gamma}_{i t}: i \in \mathcal{L}, t \in \mathcal{T}\right\}$ be an optimal solution to problem (36)-(38) and $\hat{V}_{t}\left(x_{t}\right)=\hat{\theta}_{t}+\sum_{i \in \mathcal{L}} \hat{\gamma}_{i t} x_{i t}$, we approximate the value functions $\left\{V_{t}(\cdot): t \in \mathcal{T}\right\}$ in $(2)$ by $\left\{\hat{V}_{t}(\cdot): t \in \mathcal{T}\right\}$. In this case, noting that $\hat{V}_{t+1}\left(x_{t}\right)-\hat{V}_{t+1}\left(x_{t}-\sum_{i \in \mathcal{L}} a_{i j} e_{i}\right)=\sum_{i \in \mathcal{L}} a_{i j} \hat{\gamma}_{i, t+1}$, we accept a request for itinerary $j$ at time period $t$ when we have

$$
f_{j} \geq \sum_{i \in \mathcal{L}} a_{i j} \hat{\gamma}_{i, t+1}
$$

and $a_{i j} \leq x_{i t}$ for all $i \in \mathcal{L}$. The decision rule above is similar to the one in (5) and it is equivalent to using dynamic bid prices. Adelman (2007) also shows that $V_{1}(c) \leq \hat{\theta}_{1}+\sum_{i \in \mathcal{L}} \hat{\gamma}_{i 1} c_{i}$ so that we can obtain an upper bound on the optimal total expected revenue by solving problem (36)-(38). The next proposition shows that this upper bound is tighter than the one provided by the optimal objective value of problem (13)-(16). 
Proposition 8 If $\left\{\hat{\theta}_{t}: t \in \mathcal{T}\right\}$ and $\left\{\hat{\gamma}_{i t}: i \in \mathcal{L}, t \in \mathcal{T}\right\}$ are the optimal values of the decision variables in problem (36)-(38), then we have $\hat{\theta}_{1}+\sum_{i \in \mathcal{L}} \hat{\gamma}_{i 1} c_{i} \leq \hat{\zeta}$.

Proof We let $\hat{\alpha}$ be an optimal solution to problem (6). Since we have $V_{1}^{\hat{\alpha}}(c)=\hat{\zeta}$ by Proposition 3 , we equivalently show that $\hat{\theta}_{1}+\sum_{i \in \mathcal{L}} \hat{\gamma}_{i 1} c_{i} \leq V_{1}^{\hat{\alpha}}(c)$. We let $\tilde{\theta}_{t}=\sum_{j \in \mathcal{J}} p_{j t} L_{j t}^{\hat{\alpha}}+\ldots+\sum_{j \in \mathcal{J}} p_{j \tau} L_{j \tau}^{\hat{\alpha}}$ and $\tilde{\gamma}_{i t}=r_{i t}^{\hat{\alpha}}$ for all $i \in \mathcal{L}, t \in \mathcal{T}$. By Proposition 1, we have $\tilde{\theta}_{1}+\sum_{i \in \mathcal{L}} \tilde{\gamma}_{i 1} c_{i}=\sum_{j \in \mathcal{J}} p_{j 1} L_{j 1}^{\hat{\alpha}}+$ $\ldots+\sum_{j \in \mathcal{J}} p_{j \tau} L_{j \tau}^{\hat{\alpha}}+\sum_{i \in \mathcal{L}} r_{i 1}^{\hat{\alpha}} c_{i}=V_{1}^{\hat{\alpha}}(c)$. Therefore, if we can show that the solution $\left\{\tilde{\theta}_{t}: t \in \mathcal{T}\right\}$ and $\left\{\tilde{\gamma}_{i t}: i \in \mathcal{L}, t \in \mathcal{T}\right\}$ is feasible to problem (36)-(38), then we have a feasible solution to problem (36)-(38) that yields the objective value $V_{1}^{\hat{\alpha}}(c)$ and the result follows.

For all $x_{t} \in \mathcal{C}^{|\mathcal{L}|}, u_{t} \in \mathcal{U}\left(x_{t}\right), t \in \mathcal{T} \backslash\{\tau\}$, we have

$$
\begin{aligned}
\tilde{\theta}_{t}+\sum_{i \in \mathcal{L}} \tilde{\gamma}_{i t} x_{i t} & =\sum_{j \in \mathcal{J}} p_{j t}\left[f_{j}-\sum_{i \in \mathcal{L}} a_{i j} \hat{\alpha}_{i j t}-\sum_{i \in \mathcal{L}} a_{i j} r_{i, t+1}^{\hat{\alpha}}\right]^{+}+\tilde{\theta}_{t+1}+\sum_{i \in \mathcal{L}} \sum_{j \in \mathcal{J}} p_{j t} \hat{\alpha}_{i j t} x_{i t}+\sum_{i \in \mathcal{L}} r_{i, t+1}^{\hat{\alpha}} x_{i t} \\
& \geq \sum_{j \in \mathcal{J}} p_{j t}\left[f_{j}-\sum_{i \in \mathcal{L}} a_{i j} \hat{\alpha}_{i j t}-\sum_{i \in \mathcal{L}} a_{i j} r_{i, t+1}^{\hat{\alpha}}\right] u_{j t}+\tilde{\theta}_{t+1}+\sum_{i \in \mathcal{L}} \sum_{j \in \mathcal{J}} p_{j t} \hat{\alpha}_{i j t} x_{i t}+\sum_{i \in \mathcal{L}} r_{i, t+1}^{\hat{\alpha}} x_{i t} \\
& \geq \sum_{j \in \mathcal{J}} p_{j t}\left[f_{j} u_{j t}+\tilde{\theta}_{t+1}+\sum_{i \in \mathcal{L}} r_{i, t+1}^{\hat{\alpha}}\left[x_{i t}-a_{i j} u_{j t}\right]\right]
\end{aligned}
$$

where the equality follows from the definition of $L_{j t}^{\alpha}$ and the fact that $r_{i t}^{\alpha}=\sum_{j \in \mathcal{J}} p_{j t} \alpha_{i j t}+r_{i, t+1}^{\alpha}$, the first inequality follows from the fact that $u_{j t} \in\{0,1\}$ for all $j \in \mathcal{J}$ and the second inequality follows from the fact that $a_{i j} u_{j t} \leq x_{i t}$ and $\hat{\alpha}_{i j t} \geq 0$ for all $i \in \mathcal{L}, j \in \mathcal{J}$. The last expression in the chain of inequalities above is equal to $\sum_{j \in \mathcal{J}} p_{j t}\left[f_{j} u_{j t}+\tilde{\theta}_{t+1}+\sum_{i \in \mathcal{L}} \tilde{\gamma}_{i, t+1}\left[x_{i t}-a_{i j} u_{j t}\right]\right]$, which implies that the solution $\left\{\tilde{\theta}_{t}: t \in \mathcal{T}\right\}$ and $\left\{\tilde{\gamma}_{i t}: i \in \mathcal{L}, t \in \mathcal{T}\right\}$ satisfies constraints (37). A similar argument shows that this solution also satisfies constraints (38) and the result follows.

\subsection{Capacity-Dependent Bid Prices Through linear Value Function Approximations}

Similar to the methods in Sections 4, 5.2 and 5.3, this method computes dynamic and capacity-dependent bid prices, but it builds on the optimal solution to problem (36)-(38). In particular, letting $\left\{\hat{\theta}_{t}: t \in \mathcal{T}\right\}$ and $\left\{\hat{\gamma}_{i t}: i \in \mathcal{L}, t \in \mathcal{T}\right\}$ be an optimal solution to problem (36)-(38), Zhang and Adelman (2006) propose solving the optimality equation

$$
\begin{array}{rlrl}
v_{i t}\left(x_{i t}\right)=\max & \sum_{j \in \mathcal{J}} p_{j t}\left\{\left[f_{j}-\sum_{l \in \overline{\mathcal{L}}_{i}} a_{l j} \hat{\gamma}_{l, t+1}\right] u_{j t}+v_{i, t+1}\left(x_{i t}-a_{i j} u_{j t}\right)\right\}+\sum_{l \in \overline{\mathcal{L}}_{i}}\left[\hat{\gamma}_{l t}-\hat{\gamma}_{l, t+1}\right] x_{l t} \\
\text { subject to } & a_{l j} u_{j t} \leq x_{l t} & \forall l \in \mathcal{L}, j \in \mathcal{J} \\
& u_{j t} \in\{0,1\} & \forall j \in \mathcal{J} \\
& x_{l t} \in \mathcal{C} & \forall l \in \overline{\mathcal{L}}_{i},
\end{array}
$$

where we follow the convention that $\hat{\gamma}_{i, \tau+1}=0$. In the problem above, the capacity on flight leg $i$ is the argument of the value function and it is assumed to be fixed, but the capacities on the other flight legs are decision variables. Therefore, this problem ends up being a nontrivial integer program. Zhang and Adelman (2006) show that $V_{1}(c) \leq v_{i 1}\left(c_{i}\right)+\sum_{l \in \overline{\mathcal{L}}_{i}} \hat{\gamma}_{i 1} c_{i}$ so that we can obtain an upper bound on 
the optimal total expected revenue by solving the optimality equation above. Furthermore, collecting the one-dimensional value functions $\left\{v_{i t}(\cdot): i \in \mathcal{L}, t \in \mathcal{T}\right\}$ together, we can construct the separable value function approximation $\tilde{V}_{t}\left(x_{t}\right)=\sum_{i \in \mathcal{L}} v_{i t}\left(x_{i t}\right)$ for all $t \in \mathcal{T}$. In this case, we make the itinerary acceptance decisions by replacing $\left\{V_{t}(\cdot): t \in \mathcal{T}\right\}$ on the right side of $(2)$ with $\left\{\sum_{i \in \mathcal{L}} v_{i t}(\cdot): t \in \mathcal{T}\right\}$. Similar to the methods in Sections 4, 5.2 and 5.3, it is easy to see that this idea is equivalent to using dynamic and capacity-dependent bid prices.

\section{Computational Experiments}

In this section, we compare the performances of the bid prices computed by numerous benchmark methods. Our goal is to demonstrate the benefits from using more sophisticated methods that compute dynamic and capacity-dependent bid prices.

\subsection{Benchmark Methods}

We compare the performances of the bid prices computed by the following eight benchmark methods.

Lagrangian Relaxation with Dynamic Bid Prices (LRD) This is the solution method that we develop in Sections 2 and 3, but our practical implementation recomputes the bid prices $n$ times over the planning horizon by solving problem (6) at time periods $\{1+k \tau / n: k=0,1, \ldots, n-1\}$. In particular, given the remaining leg capacities at time period $1+k \tau / n$, we solve the problem $\min _{\alpha \geq 0}\left\{V_{1+k \tau / n}^{\alpha}\left(x_{1+k \tau / n}\right)\right\}$ to obtain an optimal solution $\hat{\alpha}^{1+k \tau / n}$. We replace $\alpha$ in the decision rule in (5) with $\hat{\alpha}^{1+k \tau / n}$ and follow this decision rule until we recompute the bid prices. In all of our computational experiments, we use $n=5$ or $n=50$.

Lagrangian Relaxation with Dynamic and Capacity-Dependent Bid Prices (LRDC) This is the solution method that we develop in Section 4, but similar to LRD, our practical implementation recomputes the bid prices $n$ times over the planning horizon. In particular, given the remaining leg capacities at time period $1+k \tau / n$, we solve the problem $\min _{\alpha \geq 0}\left\{V_{1+k \tau / n}^{\alpha}\left(x_{1+k \tau / n}\right)\right\}$ to obtain an optimal solution $\hat{\alpha}^{1+k \tau / n}$. We replace $\hat{\alpha}$ in the optimality equation in (22) with $\hat{\alpha}^{1+k \tau / n}$ and solve this optimality equation to compute the value functions $\left\{v_{i t}(\cdot): i \in \mathcal{L}, t \in \mathcal{T}\right\}$. We follow the decision rule in (24) until we recompute the bid prices.

Deterministic Linear Program (DLP) This is the solution method that we describe in Section 5.1, but our practical implementation also recomputes the bid prices $n$ times over the planning horizon. In particular, given the remaining leg capacities at time period $1+k \tau / n$, we replace the right side of constraints (26) with $\left\{x_{i, 1+k \tau / n}: i \in \mathcal{L}\right\}$ and the right side of constraints $(27)$ with $\left\{\sum_{t=1+k \tau / n}^{\tau} p_{j t}\right.$ : $j \in \mathcal{J}\}$ and solve problem (25)-(28). Letting $\left\{\hat{\mu}_{i}^{1+k \tau / n}: i \in \mathcal{L}\right\}$ be the optimal values of the dual variables associated with constraints (26), we replace $\left\{\hat{\mu}_{i}: i \in \mathcal{L}\right\}$ in the decision rule in (29) with $\left\{\hat{\mu}_{i}^{1+k \tau / n}: i \in \mathcal{L}\right\}$ and follow this decision rule until we recompute the bid prices.

Randomized Linear Program (RLP) This solution method was proposed by Talluri and van Ryzin (1999). Noting that DLP uses only the expected numbers of itinerary requests, RLP tries to make up for this deficiency by working with actual samples. In particular, we let $D_{j t}$ be the number of requests 
for itinerary $j$ at time period $t$ so that we have $\mathbb{P}\left\{D_{j t}=1\right\}=p_{j t}$ and $\mathbb{P}\left\{D_{j t}=0\right\}=1-p_{j t}$. To compute the bid prices at time period $1+k \tau / n$, we generate $S$ independent samples of $D=\left\{D_{j t}: j \in \mathcal{J}, t \in \mathcal{T}\right\}$, which we denote by $\tilde{D}^{s}=\left\{\tilde{D}_{j t}^{s}: j \in \mathcal{J}, t \in \mathcal{T}\right\}$ for $s=1, \ldots, S$. Given the remaining leg capacities at time period $1+k \tau / n$, we replace the right side of constraints $(26)$ with $\left\{x_{i, 1+k \tau / n}: i \in \mathcal{L}\right\}$ and the right side of constraints (27) with $\left\{\sum_{t=1+k \tau / n}^{\tau} \tilde{D}_{j t}^{s}: j \in \mathcal{J}\right\}$ and solve problem (25)-(28). Letting $\hat{Z}^{1+k \tau / n}\left(\tilde{D}^{s}\right)$ be the optimal objective value of this problem and $\left\{\hat{\mu}_{i}^{1+k \tau / n}\left(\tilde{D}^{s}\right): i \in \mathcal{L}\right\}$ be the optimal values of the dual variables associated with constraints (26), we use $\sum_{s=1}^{S} \hat{\mu}_{i}^{1+k \tau / n}\left(\tilde{D}^{s}\right) / S$ as the bid price associated with flight leg $i$ until we recompute the bid prices.

It is also possible to show that $V_{1}(c) \leq \mathbb{E}\left\{\hat{Z}^{1}(D)\right\}$. Therefore, RLP provides an upper bound on the optimal total expected revenue, but computing $\mathbb{E}\left\{\hat{Z}^{1}(D)\right\}$ requires estimating the expectation through simulation.

Dynamic Programming Decomposition (DPD) This is the solution method that we describe in Section 5.2 , but our practical implementation recomputes the bid prices $n$ times over the planning horizon by using an approach similar to the one used by LRDC.

Decomposition by Revenue Allocation (DRA) This is the solution method that we describe in Section 5.3 , but similar to LRDC and DPD, our practical implementation recomputes the bid prices $n$ times over the planning horizon. In particular, given the remaining leg capacities at time period $1+k \tau / n$,

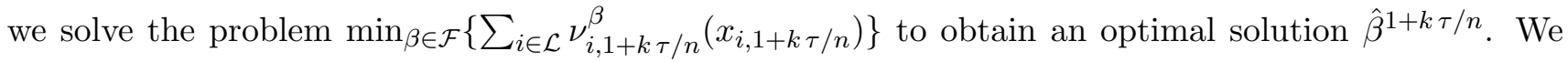
replace $\hat{\beta}$ in the decision rule in (35) with $\hat{\beta}^{1+k \tau / n}$ and follow this decision rule until we recompute the bid prices.

Linear Approximations with Dynamic Bid Prices (LAD) This is the solution method that we describe in Section 5.4, but similar to LRD and DLP, our practical implementation recomputes the bid prices $n$ times over the planning horizon. In particular, given the remaining leg capacities at time period $1+k \tau / n$, we replace the objective function of problem (36)-(38) with $\theta_{1+k \tau / n}+\sum_{i \in \mathcal{L}} \gamma_{i, 1+k \tau / n} x_{i, 1+k \tau / n}$ and solve this problem to obtain an optimal solution $\left\{\hat{\theta}_{t}^{1+k \tau / n}: t \in \mathcal{T}\right\}$ and $\left\{\hat{\gamma}_{i t}^{1+k \tau / n}: i \in \mathcal{L}, t \in \mathcal{T}\right\}$. We replace $\left\{\hat{\gamma}_{i t}: i \in \mathcal{L}, t \in \mathcal{T}\right\}$ in the decision rule in $(39)$ with $\left\{\hat{\gamma}_{i t}^{1+k \tau / n}: i \in \mathcal{L}, t \in \mathcal{T}\right\}$ and follow this decision rule until we recompute the bid prices.

Linear Approximations with Dynamic and Capacity-Dependent Bid Prices (LADC) This is the solution method that we describe in Section 5.5, but our practical implementation recomputes the bid prices $n$ times over the planning horizon by using an approach similar to the one used by LRDC.

\subsection{EXPERIMENTAL Setup}

We consider two types of airline networks in our computational experiments. The first airline network includes one hub and $N$ spokes. There are two flight legs associated with each spoke. One of these flight legs is from the spoke to the hub and the other one is from the hub to the spoke. There is a high-fare and a low-fare itinerary that connect each possible origin-destination pair. Therefore, the first airline network includes $2 N$ flight legs and $2 N(N+1)$ itineraries, and an itinerary includes at most two flight legs. The structure of the first airline network for $N=6$ is shown on the left side of Figure 1. 
The second airline network includes two hubs and $N$ spokes. The first half of the spokes are connected to the first hub and the second half of the spokes are connected to the second hub. There are two flight legs associated with each spoke. One of these flight legs is from the spoke to the hub and the other one is from the hub to the spoke. There are also two flight legs that connect the hubs in each direction. We sample 50 to 150 origin-destination pairs among the set of all possible origin-destination pairs and assume that there is a high-fare and a low-fare itinerary that connect each sampled origin-destination pair. Therefore, the second airline network includes $2 N+2$ flights and 100 to 300 itineraries, and an itinerary includes at most three flight legs. The structure of the second airline network for $N=10$ is shown on the right side of Figure 1.

For both airline networks, the revenue associated with a high-fare itinerary is $\kappa$ times larger than the revenue associated with the corresponding low-fare itinerary. We generate the arrival probabilities $\left\{p_{j t}: j \in \mathcal{J}, t \in \mathcal{T}\right\}$ in such a manner that the probability of having a request for a high-fare itinerary increases over time, whereas the probability of having a request for a low-fare itinerary decreases over time. To do this, we randomly generate one probability for each origin-destination pair in the airline network such that the sum of these probabilities over all origin-destination pairs is equal to 1 . If we let $P_{o d}$ be the probability that we generate for origin-destination pair $(o, d)$ and $\lambda(t)$ be an increasing function of $t$ taking values over $[0,1]$, then the probability that there is a request at time period $t$ for the high-fare itinerary associated with origin-destination pair $(o, d)$ is $\lambda(t) P_{o d}$ and the probability that there is a request at time period $t$ for the low-fare itinerary associated with origin-destination pair $(o, d)$ is $[1-\lambda(t)] P_{o d}$. In our test problems, we have $\lambda(t)=0$ until about one third of the planning horizon, and after this, $\lambda(t)$ increases linearly to 1 . Since the total expected demand for the capacity on flight leg $i$ is $\sum_{t \in \mathcal{T}} \sum_{j \in \mathcal{J}} a_{i j} p_{j t}$, we measure the tightness of the leg capacities by

$$
\theta=\frac{\sum_{t \in \mathcal{T}} \sum_{j \in \mathcal{J}} \sum_{i \in \mathcal{L}} a_{i j} p_{j t}}{\sum_{i \in \mathcal{L}} c_{i}} .
$$

We vary $N, \theta$ and $\kappa$ in our computational experiments and label our test problems by $(N, \theta, \kappa)$.

Section 3 shows that we can obtain an optimal solution to the problem $\min _{\alpha \geq 0}\left\{V_{1+k \tau / n}^{\alpha}\left(x_{1+k \tau / n}\right)\right\}$ by solving a linear program. We use $S=50$ for RLP. We use subgradient optimization to solve the

problem $\min _{\beta \in \mathcal{F}}\left\{\sum_{i \in \mathcal{L}} \nu_{i, 1+k \tau / n}^{\beta}\left(x_{i, 1+k \tau / n}\right)\right\}$ for DRA. The step size and termination criterion that we use for subgradient optimization are the same as those in Topaloglu (2009). When recomputing the bid prices for LAD, we solve the dual of problem (36)-(38) through column generation with $5 \%$ optimality gap. However, when computing the upper bounds on the optimal total expected revenue, we solve problem (36)-(38) to optimality so that the upper bounds that we report are accurate.

\subsection{Computational Results for the First Airline Network}

Tables 1 and 2 show the total expected revenues obtained by the different benchmark methods for the first airline network. Table 1 corresponds to the case where we recompute the bid prices five times over the planning horizon, whereas Table 2 corresponds to the case where we recompute the bid prices 50 times. The first column in these tables shows the characteristics of the test problems. The next eight columns show the total expected revenues obtained by LRD, LRDC, DLP, RLP, DPD, DRA, LAD 
and LADC. We estimate these total expected revenues by simulating the performances of the different benchmark methods under multiple demand trajectories. To reduce the effect of simulation noise, we use common random numbers when simulating the performances of the different benchmark methods; see Law and Kelton (2000). The tenth column shows the percent gap between the total expected revenues obtained by LRDC and LRD. This column also includes a " $\checkmark$ " whenever LRDC performs better than LRD, a " $\times$ " whenever LRDC performs worse than LRD and a " $\odot$ " whenever there is no statistically significant difference between the total expected revenues obtained by LRDC and LRD at $95 \%$ level. The last six columns do the same thing as the tenth column, but they compare the performance of LRDC with the remaining six benchmark methods. LRDC turns out to be one of the better benchmark methods and we use it as a reference point.

The results indicate that LRDC performs noticeably better than the other benchmark methods. When we recompute the bid prices five times over the planning horizon, LRDC improves on LRD, DLP, RLP and LAD respectively by $5.6 \%, 7.5 \% 3.9 \%$ and $5.0 \%$ on the average. The same improvement figures decrease respectively to $2.5 \%, 4.1 \%, 1.5 \%$ and $2.9 \%$ when we recompute the bid prices 50 times. In either case, such improvement figures are considered quite significant in the network revenue management setting. The improvements of LRDC over LRD, DLP, RLP and LAD are most noticeable when the expected demand exceeds the capacity by a large margin and there is a large gap between the revenues associated with the high-fare and low-fare itineraries. Therefore, it is important to use a more sophisticated method to compute the bid prices when the leg capacities are scarce and there is substantial regret associated with accepting a low-fare itinerary request when one could have accepted a high-fare itinerary request.

LRD consistently performs better than DLP. This is due to the fact that problem (13)-(16) captures the dynamics of the network revenue management problem better than problem (25)-(28). On the other hand, randomization substantially improves the performance of the bid prices computed by problem (25)-(28) and RLP performs better than LRD and DLP for a majority of the test problems. Although both LRD and LAD compute dynamic bid prices, LAD has a slight advantage over LRD for a majority of the test problems.

The performances of LRDC, DPD, DRA and LADC are comparable. LRDC performs better than DPD and DRA by a small but consistent margin when we recompute the bid prices five times over the planning horizon. In this case, the total expected revenues obtained by LRDC are either better than or indistinguishable from those obtained by DPD and DRA. It turns out that DRA catches up with LRDC when we recompute the bid prices 50 times. On the other hand, LRDC generally performs better than DPD irrespective of how frequently we recompute the bid prices. This is expected since LRDC builds on the optimal dual solution to problem (13)-(16), whereas DPD builds on the optimal dual solution to problem (25)-(28) and problem (13)-(16) captures the capacity availabilities better than problem (25)-(28). The performances of LRDC and LADC are quite close. We note that LADC builds on the optimal solution to problem (36)-(38) and this problem provides a tighter upper bound on the optimal total expected revenue than problem (13)-(16). However, this potential advantage does not seem to help LADC too much and its performance is quite close to that of LRDC. 
Figure 2 shows the improvements in the performances of the different benchmark methods when we recompute the bid prices 50 times over the planning horizon instead of five times. LRD, DLP, RLP and LAD significantly benefit from recomputing the bid prices more frequently. Recomputing the bid prices more frequently also helps the performance of DRA, but not as much as it helps the performances of LRD, DLP, RLP and LAD. On the other hand, LRDC, DPD and LADC do not benefit much from recomputing the bid prices more frequently. This observation can be explained by the fact that the decision rules used by LRD, DLP, RLP and LAD are equivalent to approximating the value functions by linear functions and the linear value function approximations tend to be accurate only around the current values of the remaining leg capacities, but not necessarily around the future values of the remaining leg capacities. In other words, the value function approximations used by LRD, DLP, RLP and LAD do not capture the curvature of the value functions and they need to be retuned frequently as the values of the remaining leg capacities change. On the other hand, the decision rules used by LRDC, DPD, DRA and LADC are equivalent to approximating the value functions by separable concave functions and these value function approximations capture the curvature of the value functions, at least to a certain extent. It is also interesting to note that there are quite a few test problems where the performances of LRDC, DPD and LADC slightly deteriorate when we recompute the bid prices more frequently. Similar behavior for other methods in the literature is analyzed in Cooper (2002).

Table 3 shows the CPU seconds required by the different benchmark methods to compute one set of bid prices. All of our computational experiments are run on a Pentium IV PC running Windows XP with $2.4 \mathrm{GHz}$ CPU and $1 \mathrm{~GB}$ RAM. The runtimes for LAD and LADC correspond to the case where we solve problem (36)-(38) with $5 \%$ optimality gap. The results indicate that the runtimes for DLP, RLP and DPD are quite short. The runtimes for LRD are longer, but they are under two seconds for a majority of the test problems. The runtimes for LRD and LRDC differ by about one second. The runtimes for DRA exceed those for the other benchmark methods by orders of magnitude. Although DRA performs quite well when we recompute the bid prices 50 times over the planning horizon, this comes at the cost of a significant increase in the runtimes. The runtimes for LAD and LADC are also significantly longer than those for LRD and LRDC. Therefore, LRDC and DPD seem to strike a reasonable balance between performance and computational burden, though LRDC has a small but consistent performance advantage over DPD.

Table 4 shows the upper bounds on the optimal total expected revenues provided by the different benchmark methods. The columns in this table have the same interpretations as those in Tables 1 and 2 , but they compare the upper bounds instead of the total expected revenues. The results indicate that DRA consistently provides the tightest upper bounds. The upper bounds provided by LRDC, RLP and LADC compete for the second place, but we emphasize that the upper bounds provided by RLP require estimating the expectation $\mathbb{E}\left\{\hat{Z}^{1}(D)\right\}$ through simulation, whereas the upper bounds provided by LRDC and LADC can be computed exactly. The upper bounds provided by LRD, DPD and LAD compete for the fifth place. DLP consistently provides the loosest upper bounds and this is in agreement with Propositions 5 and 7. One interesting observation is that LRD and LAD provide the same upper bounds for all of our test problems. Proposition 8 shows that the upper bound provided by LAD is potentially tighter than the one provided by LRD, but these upper bounds coincide for all of our test 
problems. We believe that this one of the reasons why LADC does not perform better than LRDC, although it is based on a linear program that potentially provides tighter upper bounds.

\subsection{Computational Results for the Second Airline Network}

Tables 5 and 6 show the total expected revenues obtained by the different benchmark methods for the second airline network. Table 5 corresponds to the case where we recompute the bid prices five times over the planning horizon, whereas Table 6 corresponds to the case where we recompute the bid prices 50 times. The columns in these tables have the same interpretations as those in Tables 1 and 2. For a majority of the test problems that take place over the second airline network, DRA requires more than 1 GB RAM and we do not use DRA as a benchmark method for the second airline network.

The results display essentially the same trends as those in Tables 1 and 2. When we recompute the bid prices five times over the planning horizon, LRDC improves on LRD, DLP, RLP and LAD respectively by $4.1 \%, 5.5 \%, 3.2 \%$ and $3.9 \%$ on the average. The same improvement figures decrease to $1.9 \%, 3.1 \%, 1.2 \%$ and $2.5 \%$ when we recompute the bid prices 50 times. LRDC generally performs better than DPD by a small but consistent margin. The performance gaps between LRDC and DPD are statistically significant for a majority of the test problems. The performance of LRDC is essentially indistinguishable from that of LADC.

Table 7 shows the CPU seconds required by the different benchmark methods to compute one set of bid prices. The runtimes for LRD are under six seconds and the runtimes for LRDC are under 10 seconds for a majority of the test problems. The runtimes for DPD are about twice as fast as those for LRDC. The runtimes for LAD and LADC approach one minute for the test problems with large numbers of spokes. Noting that LRDC performs essentially the same as LADC, LRDC appears to be an appealing alternative to LADC.

Table 8 shows the upper bounds on the optimal total expected revenues provided by the different benchmark methods. The columns in this table have the same interpretations as those in Table 4. The results indicate that LRDC, RLP and LADC provide the tightest upper bounds. The upper bounds provided by LRDC and LADC are tighter than those provided by RLP when the expected demand exceeds the capacity by a large margin. The upper bounds provided by LRD and LAD once again coincide for all of our test problems.

\section{Conclusions}

We presented a new method to compute bid prices in network revenue management problems. The novel aspect of our method is that it naturally provides dynamic bid prices that depend on how much time is left until departure. Our method provides an upper bound on the optimal total expected revenue and this upper bound is tighter than the one provided by the deterministic linear program. The bid prices computed by our method can be used in a dynamic programming decomposition-like idea to decompose the network revenue management problem by the flight legs and to obtain dynamic and capacity-dependent bid prices. 
Our computational experiments indicated that dynamic and capacity-dependent bid prices perform quite well. In particular, the four benchmark methods, LRDC, DPD, DRA and LADC, which compute dynamic and capacity-dependent bid prices, perform substantially better than LRD, DLP, RLP and LAD. Comparing LRDC, DPD, DRA and LADC among each other, DRA is comparable to LRDC when we recompute the bid prices frequently, but it has a higher computational burden. On the other hand, LRDC performs better than DPD by a small but consistent margin. The performance gaps between LRDC and DPD are statistically significant for many test problems and these performance gaps can approach $1 \%$ for the test problems with large numbers of spokes. The performances of LRDC and LADC are essentially indistinguishable, but the runtimes for LADC are significantly longer than those for LRDC. Therefore, LRDC, which uses the bid prices computed by our method as a starting point, seems to strike a reasonable balance between performance and computational burden.

\section{REFERENCES}

Adelman, D. (2007), 'Dynamic bid-prices in revenue management', Operations Research 55(4), 647-661.

Adelman, D. and Mersereau, A. J. (2008), 'Relaxations of weakly coupled stochastic dynamic programs', Operations Research 56(3), 712-727.

Belobaba, P. P. (1987), Air Travel Demand and Airline Seat Inventory Control, PhD thesis, Massachusetts Institute of Technology, Cambridge, MA.

Bertsekas, D. P. (2001), Dynamic Programming and Optimal Control, Athena Scientific, Belmont, MA.

Bertsimas, D. and Popescu, I. (2003), 'Revenue management in a dynamic network environment', Transportation Science 37, 257-277.

Castanon, D. A. (1997), Approximate dynamic programming for sensor management, in 'Proceedings of the 36th Conference on Decision \& Control'.

Cheung, R. K. and Powell, W. B. (1996), 'An algorithm for multistage dynamic networks with random arc capacities, with an application to dynamic fleet management', Operations Research 44(6), 951963.

Cooper, W. L. (2002), 'Asymptotic behavior of an allocation policy for revenue management', Operations Research 50(4), 720-727.

Gallego, G., Iyengar, G., Phillips, R. and Dubey, A. (2004), Managing flexible products on a network, CORC Technical Report TR-2004-01, Columbia University.

Hawkins, J. (2003), A Lagrangian Decomposition Approach to Weakly Coupled Dynamic Optimization Problems and its Applications, PhD thesis, Massachusetts Institute of Technology, Cambridge, MA.

Karmarkar, U. S. (1981), 'The multiperiod multilocation inventory problems', Operations Research 29, 215-228.

Kunnumkal, S. and Topaloglu, H. (2007), A new dynamic programming decomposition method for the network revenue management problem with customer choice behavior, Technical report, Cornell University, School of Operations Research and Information Engineering. Available at http://legacy.orie.cornell.edu/ huseyin/publications/publications.html.

Kunnumkal, S. and Topaloglu, H. (2008a), 'A duality-based relaxation and decomposition approach for inventory distribution systems', Naval Research Logistics Quarterly 55(7), 612-631.

Kunnumkal, S. and Topaloglu, H. (2008b), 'A refined deterministic linear program for the network revenue management problem with customer choice behavior', Naval Research Logistics Quarterly $\mathbf{5 5}, 563-580$. 
Law, A. L. and Kelton, W. D. (2000), Simulation Modeling and Analysis, McGraw-Hill, Boston, MA.

Liu, Q. and van Ryzin, G. (2008), 'On the choice-based linear programming model for network revenue management', Manufacturing 85 Service Operations Management 10(2), 288-310.

Ruszczynski, A. (2003), Decomposition methods, in A. Ruszczynski and A. Shapiro, eds, 'Handbook in Operations Research and Management Science, Volume on Stochastic Programming', North Holland, Amsterdam.

Simpson, R. W. (1989), Using network flow techniques to find shadow prices for market and seat inventory control, Technical report, MIT Flight Transportation Laboratory Memorandum M89-1, Cambridge, MA.

Talluri, K. T. and van Ryzin, G. J. (2004), The Theory and Practice of Revenue Management, Kluwer Academic Publishers.

Talluri, K. and van Ryzin, G. (1998), 'An analysis of bid-price controls for network revenue management', Management Science 44(11), 1577-1593.

Talluri, K. and van Ryzin, G. (1999), 'A randomized linear programming method for computing network bid prices', Transportation Science 33(2), 207-216.

Topaloglu, H. (2009), 'Using Lagrangian relaxation to compute capacity-dependent bid-prices in network revenue management', Operations Research 57(3), 637-649.

Topaloglu, H. and Kunnumkal, S. (2006), Computing time-dependent bid prices in network revenue management problems, Technical report, Cornell University, School of Operations Research and Information Engineering.

Available at http://legacy.orie.cornell.edu/ huseyin/publications/publications.html.

Varaiya, P. P. (1998), 'Lecture notes on optimization', Unpublished manuscript, University of California, Department of Electrical Engineering and Computer Science.

Williamson, E. L. (1992), Airline Network Seat Control, PhD thesis, Massachusetts Institute of Technology, Cambridge, MA.

Wolsey, L. A. (1998), Integer Programming, John Wiley \& Sons, Inc., New York.

Zhang, D. and Adelman, D. (2006), An approximate dynamic programming approach to network revenue management with customer choice, Technical report, University of Chicago, Graduate School of Business. 

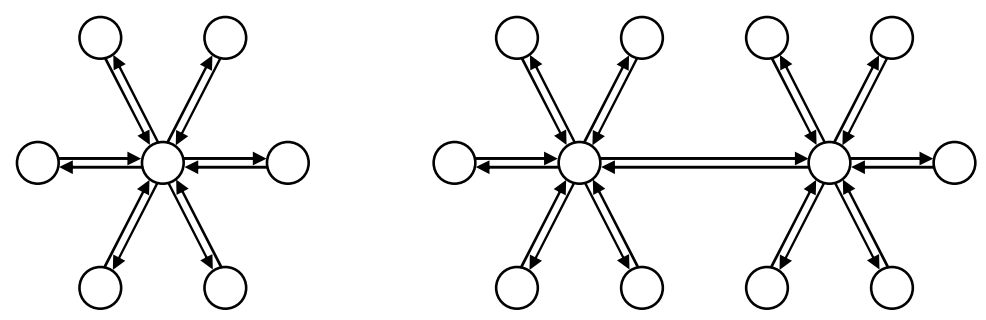

Figure 1: Structures of the two airline networks that we use in our computational experiments.

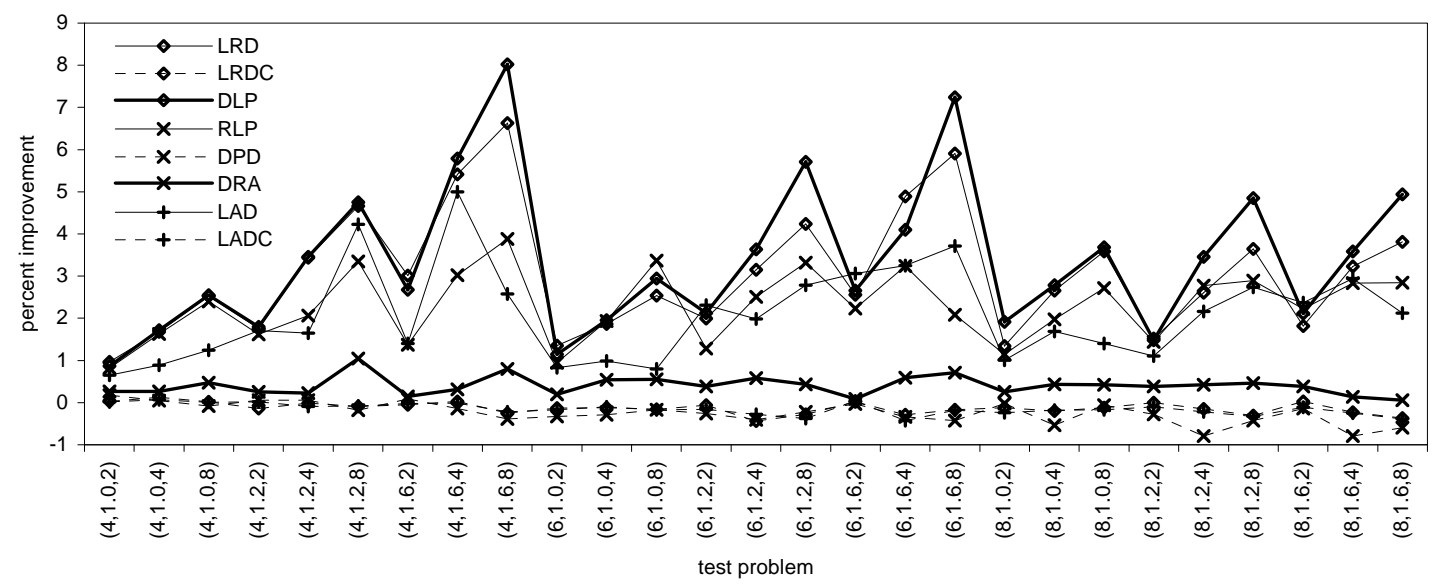

Figure 2: Improvements in the performances of the benchmark methods for the first airline network when we recompute the bid prices 50 times over the planning horizon instead of five times. 


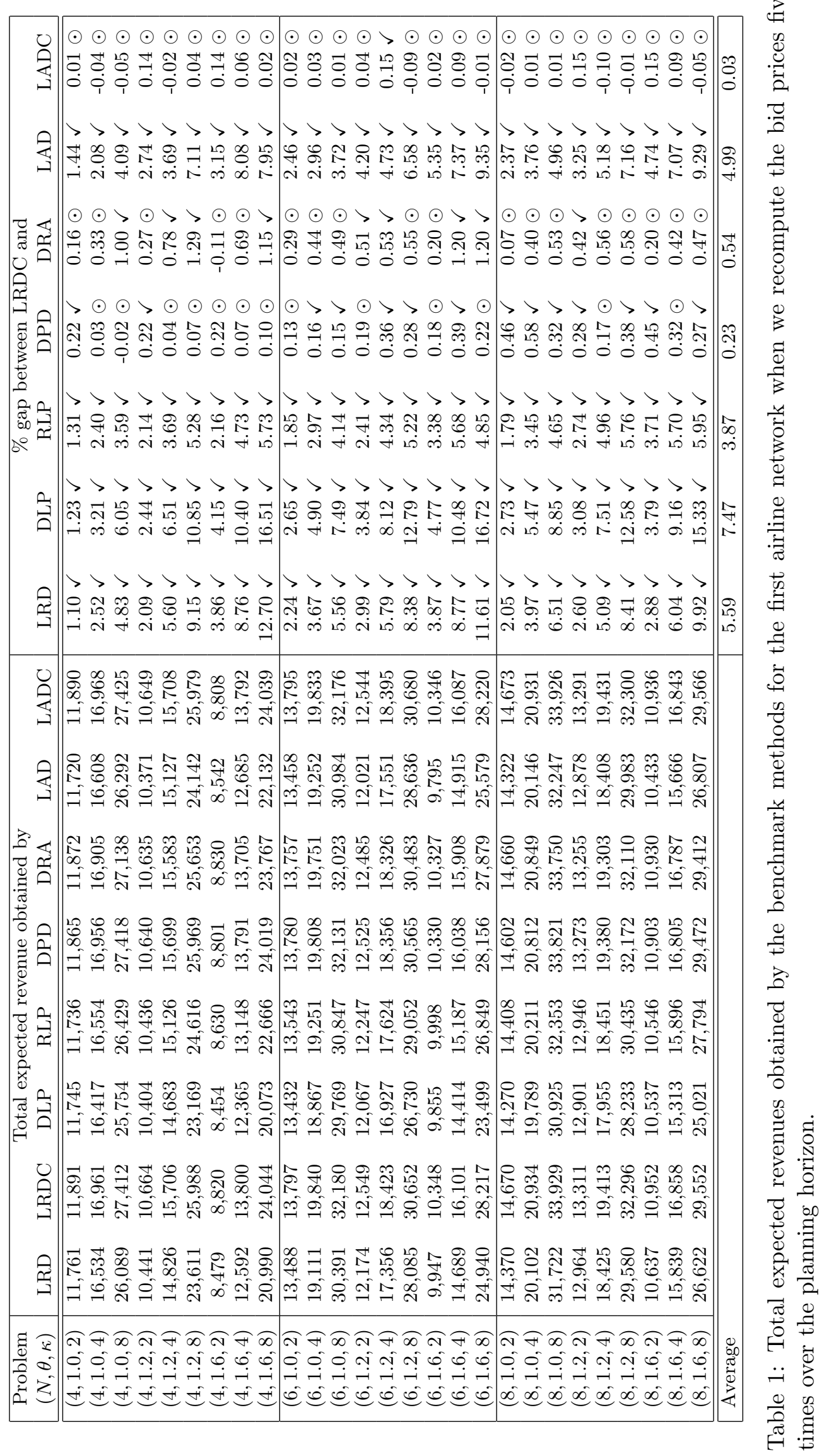




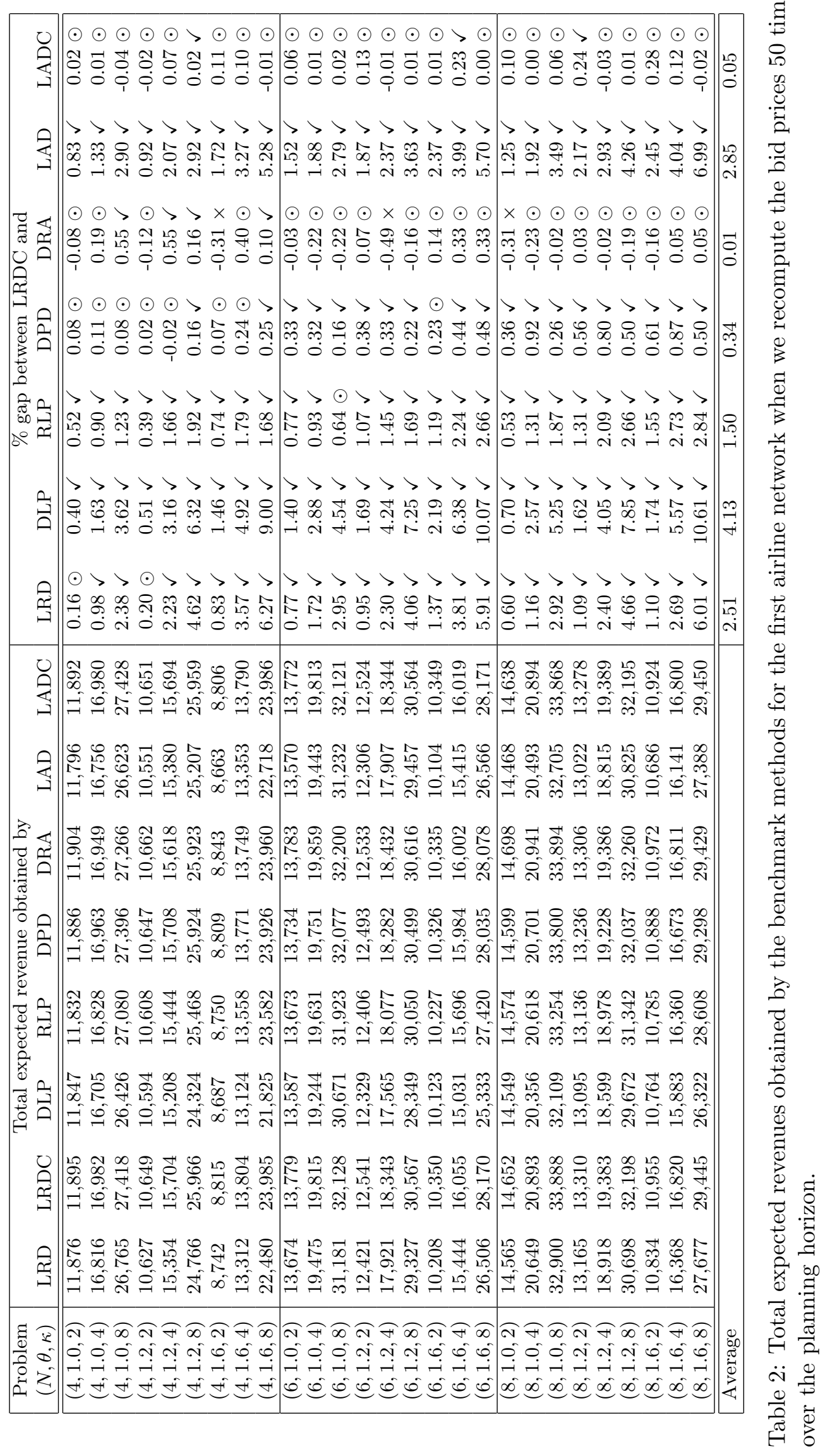




\begin{tabular}{|c|cccccccc|}
\hline Problem & & & \multicolumn{7}{c|}{ CPU seconds for } \\
$(N, \theta, \kappa)$ & LRD & LRDC & DLP & RLP & DPD & DRA & LAD & LADC \\
\hline \hline$(4,1.0,2)$ & 0.31 & 0.81 & 0.00 & 0.03 & 0.42 & 76.38 & 6.25 & 7.01 \\
$(4,1.0,4)$ & 0.24 & 0.81 & 0.02 & 0.05 & 0.42 & 39.94 & 6.72 & 6.92 \\
$(4,1.0,8)$ & 0.23 & 0.82 & 0.02 & 0.05 & 0.42 & 13.63 & 6.42 & 7.58 \\
$(4,1.2,2)$ & 0.33 & 0.80 & 0.02 & 0.05 & 0.34 & 30.56 & 8.13 & 8.66 \\
$(4,1.2,4)$ & 0.24 & 0.73 & 0.00 & 0.05 & 0.34 & 57.81 & 7.20 & 8.30 \\
$(4,1.2,8)$ & 0.24 & 0.72 & 0.00 & 0.05 & 0.36 & 60.47 & 7.50 & 7.75 \\
$(4,1.6,2)$ & 0.41 & 0.77 & 0.00 & 0.05 & 0.28 & 44.11 & 8.81 & 9.26 \\
$(4,1.6,4)$ & 0.26 & 0.63 & 0.00 & 0.05 & 0.28 & 32.13 & 8.71 & 8.95 \\
$(4,1.6,8)$ & 0.24 & 0.61 & 0.00 & 0.05 & 0.28 & 51.31 & 8.17 & 8.80 \\
\hline$(6,1.0,2)$ & 1.23 & 2.53 & 0.00 & 0.06 & 0.92 & 52.19 & 15.58 & 17.05 \\
$(6,1.0,4)$ & 1.09 & 2.41 & 0.00 & 0.06 & 0.92 & 34.02 & 13.74 & 16.39 \\
$(6,1.0,8)$ & 1.06 & 2.37 & 0.00 & 0.06 & 0.92 & 33.45 & 16.60 & 17.89 \\
$(6,1.2,2)$ & 1.81 & 2.87 & 0.00 & 0.06 & 0.78 & 82.09 & 24.31 & 25.67 \\
$(6,1.2,4)$ & 1.30 & 2.41 & 0.00 & 0.06 & 0.78 & 234.86 & 30.28 & 32.50 \\
$(6,1.2,8)$ & 1.13 & 2.22 & 0.00 & 0.06 & 0.78 & 231.20 & 26.45 & 27.63 \\
$(6,1.6,2)$ & 3.12 & 3.87 & 0.00 & 0.06 & 0.59 & 45.28 & 27.81 & 28.59 \\
$(6,1.6,4)$ & 1.65 & 2.49 & 0.00 & 0.08 & 0.59 & 165.77 & 33.52 & 35.25 \\
$(6,1.6,8)$ & 1.21 & 2.06 & 0.00 & 0.06 & 0.59 & 119.44 & 29.05 & 30.86 \\
\hline$(8,1.0,2)$ & 1.02 & 3.30 & 0.02 & 0.09 & 1.66 & 144.61 & 33.09 & 36.66 \\
$(8,1.0,4)$ & 0.60 & 2.91 & 0.00 & 0.09 & 1.63 & 360.86 & 36.95 & 39.55 \\
$(8,1.0,8)$ & 0.47 & 2.81 & 0.02 & 0.09 & 1.63 & 307.61 & 30.69 & 33.64 \\
$(8,1.2,2)$ & 3.30 & 5.19 & 0.00 & 0.11 & 1.39 & 129.72 & 64.06 & 66.72 \\
$(8,1.2,4)$ & 1.00 & 2.95 & 0.02 & 0.11 & 1.39 & 284.78 & 57.49 & 59.91 \\
$(8,1.2,8)$ & 0.54 & 2.55 & 0.02 & 0.09 & 1.41 & 206.91 & 52.33 & 54.95 \\
$(8,1.6,2)$ & 6.63 & 8.04 & 0.02 & 0.13 & 1.08 & 137.77 & 66.91 & 68.89 \\
$(8,1.6,4)$ & 1.62 & 3.10 & 0.00 & 0.09 & 1.05 & 170.36 & 60.81 & 63.69 \\
$(8,1.6,8)$ & 0.69 & 2.17 & 0.02 & 0.11 & 1.06 & 470.28 & 55.16 & 57.58 \\
\hline
\end{tabular}

Table 3: CPU seconds required by the benchmark methods to compute one set of bid prices for the first airline network. The runtimes that are less than 5 milliseconds are indicated as zero. 


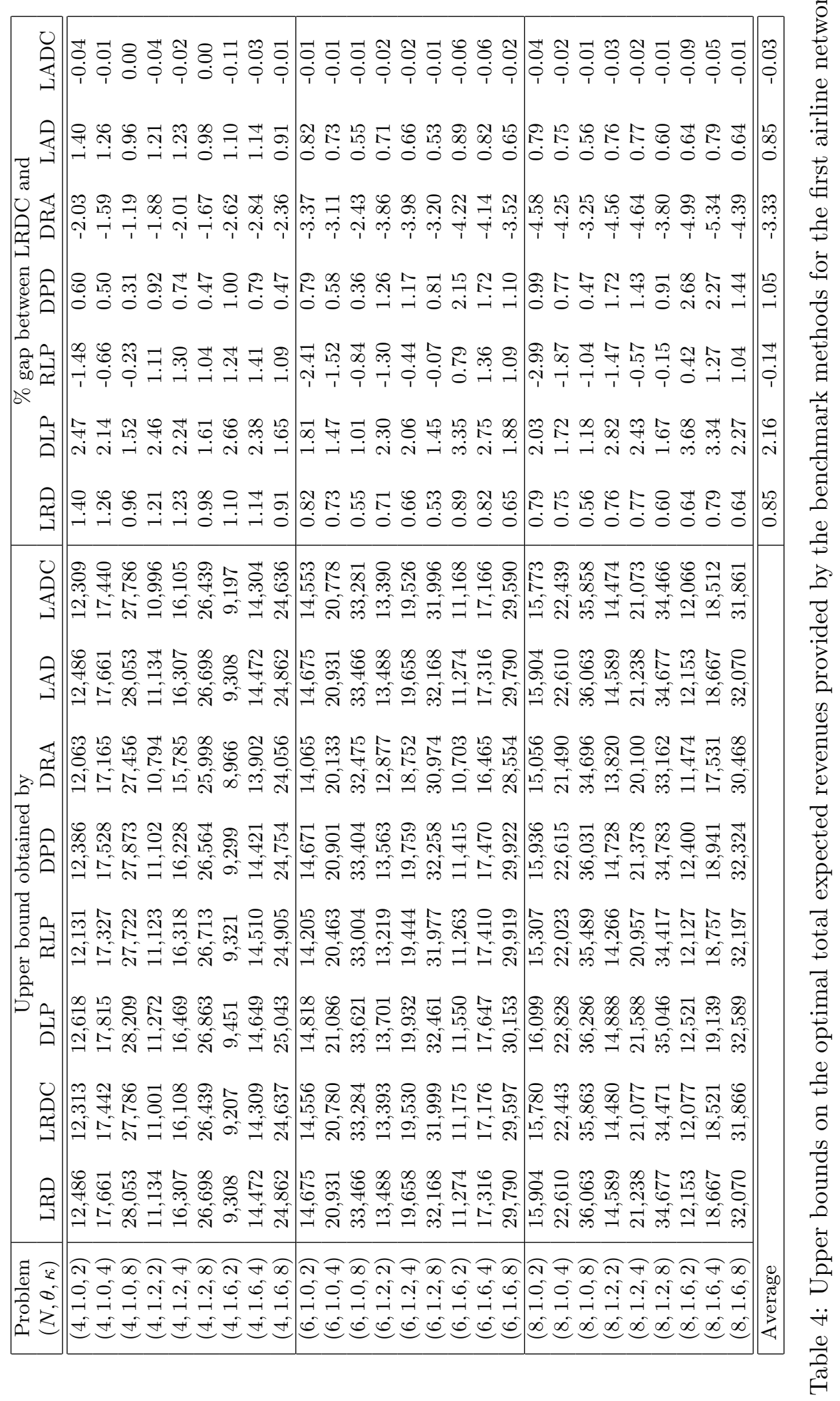




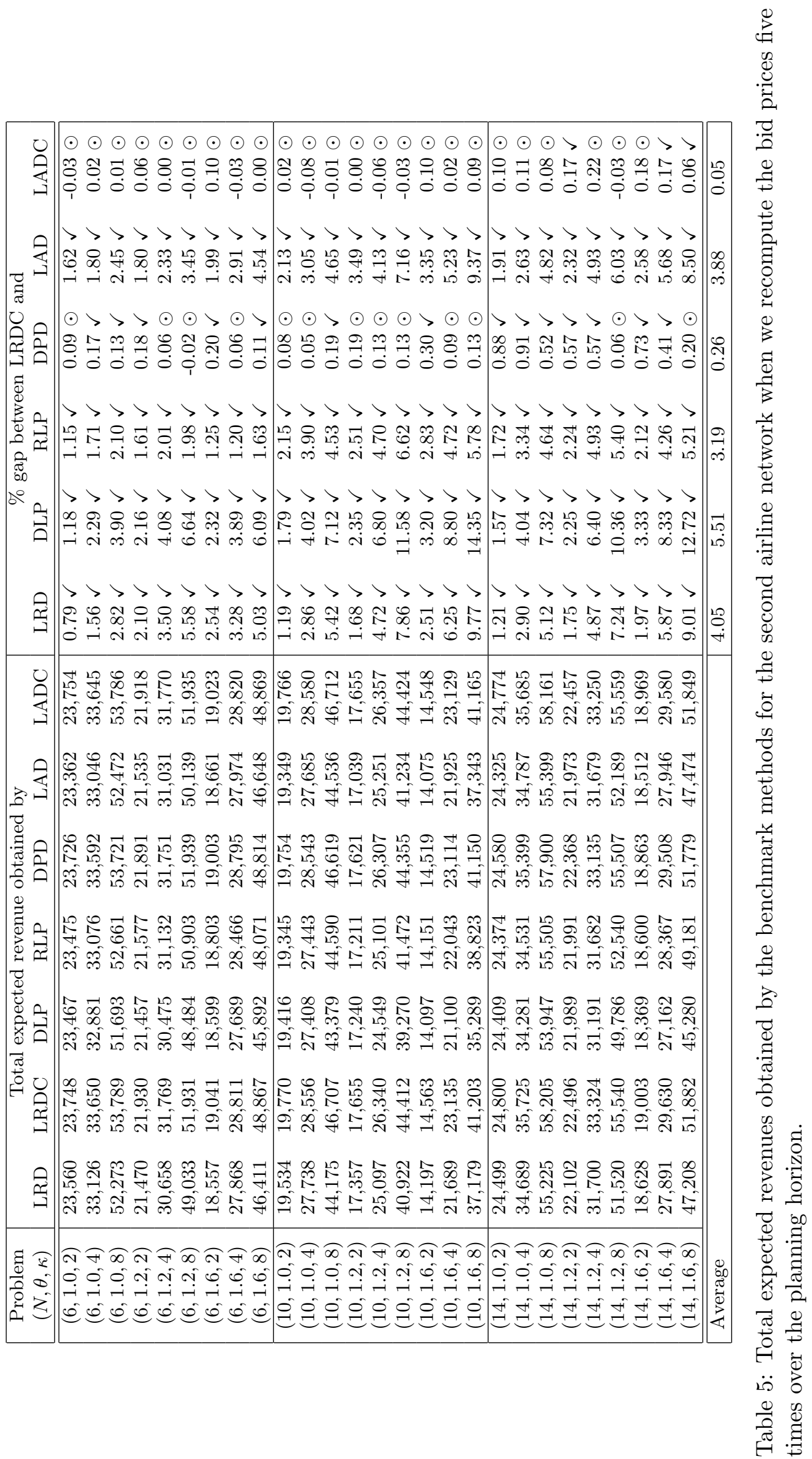




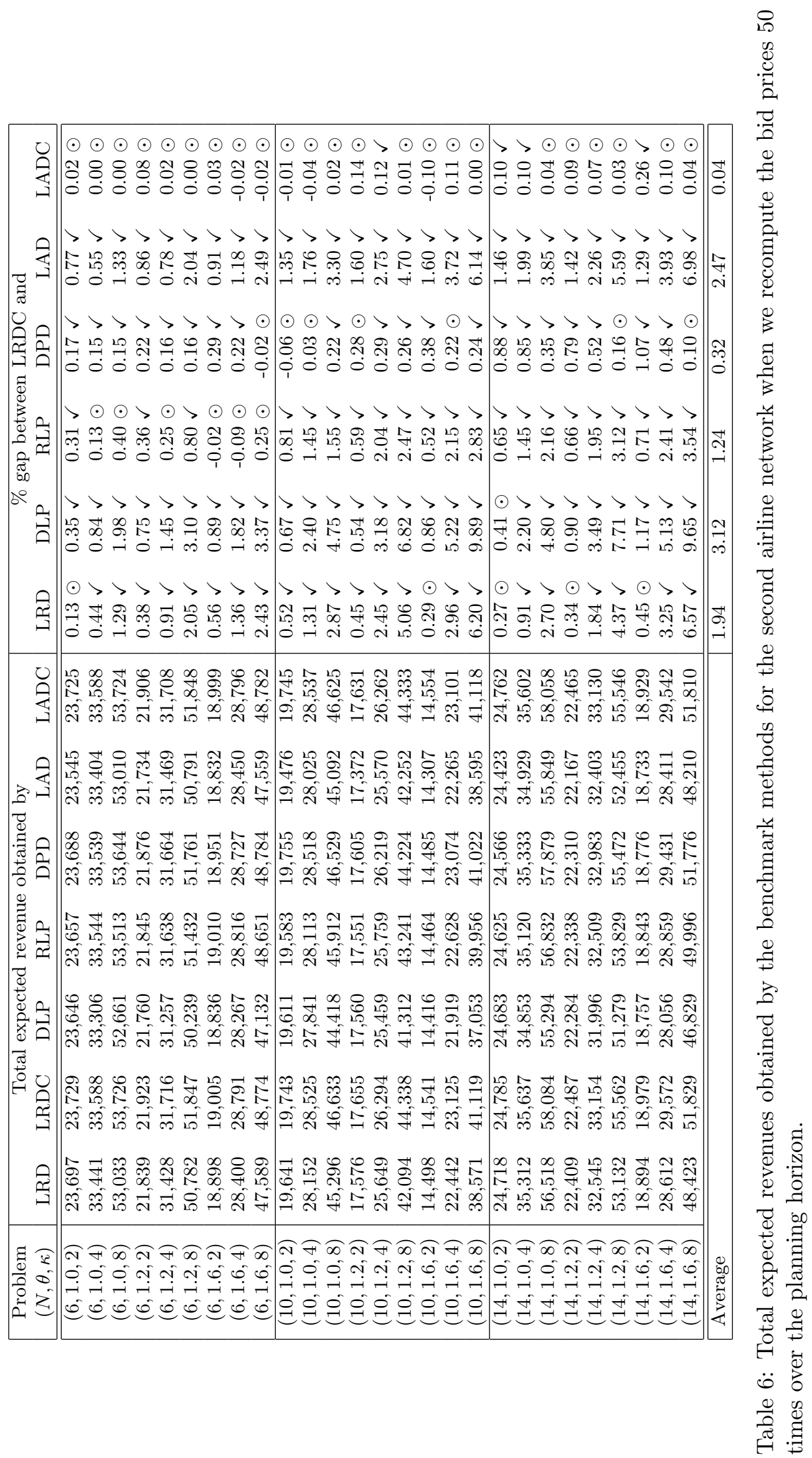




\begin{tabular}{|c|ccccccc|}
\hline Problem & \multicolumn{7}{|c|}{ CPU seconds for } \\
$(N, \theta, \kappa)$ & LRD & LRDC & DLP & RLP & DPD & LAD & LADC \\
\hline \hline$(6,1.0,2)$ & 0.78 & 2.51 & 0.00 & 0.05 & 1.38 & 2.39 & 3.80 \\
$(6,1.0,4)$ & 0.75 & 2.21 & 0.00 & 0.05 & 1.38 & 2.74 & 4.08 \\
$(6,1.0,8)$ & 0.75 & 2.43 & 0.00 & 0.05 & 1.38 & 2.52 & 3.80 \\
$(6,1.2,2)$ & 0.77 & 2.29 & 0.00 & 0.05 & 1.16 & 6.59 & 7.72 \\
$(6,1.2,4)$ & 0.75 & 1.96 & 0.00 & 0.05 & 1.16 & 6.78 & 7.60 \\
$(6,1.2,8)$ & 0.84 & 1.68 & 0.00 & 0.05 & 1.16 & 6.89 & 7.73 \\
$(6,1.6,2)$ & 0.75 & 1.66 & 0.00 & 0.05 & 0.88 & 7.00 & 7.81 \\
$(6,1.6,4)$ & 0.75 & 1.67 & 0.00 & 0.05 & 0.88 & 5.77 & 6.61 \\
$(6,1.6,8)$ & 0.75 & 1.66 & 0.00 & 0.05 & 0.88 & 5.69 & 6.69 \\
\hline$(10,1.0,2)$ & 5.31 & 8.87 & 0.00 & 0.05 & 3.27 & 12.25 & 15.41 \\
$(10,1.0,4)$ & 5.09 & 8.56 & 0.02 & 0.05 & 3.31 & 14.89 & 17.86 \\
$(10,1.0,8)$ & 4.98 & 8.65 & 0.02 & 0.05 & 3.31 & 14.73 & 17.77 \\
$(10,1.2,2)$ & 5.56 & 8.62 & 0.00 & 0.05 & 2.75 & 23.66 & 26.91 \\
$(10,1.2,4)$ & 5.14 & 8.14 & 0.02 & 0.05 & 2.75 & 22.27 & 23.98 \\
$(10,1.2,8)$ & 5.02 & 8.54 & 0.02 & 0.06 & 2.78 & 22.45 & 24.86 \\
$(10,1.6,2)$ & 5.74 & 8.05 & 0.02 & 0.05 & 2.11 & 21.64 & 24.02 \\
$(10,1.6,4)$ & 5.23 & 7.57 & 0.00 & 0.06 & 2.13 & 22.47 & 26.63 \\
$(10,1.6,8)$ & 5.03 & 7.88 & 0.00 & 0.06 & 2.09 & 22.22 & 25.00 \\
\hline$(14,1.0,2)$ & 4.65 & 11.92 & 0.03 & 0.06 & 5.92 & 42.13 & 51.16 \\
$(14,1.0,4)$ & 3.29 & 10.23 & 0.03 & 0.06 & 5.94 & 38.64 & 45.77 \\
$(14,1.0,8)$ & 3.16 & 9.94 & 0.03 & 0.06 & 5.94 & 42.16 & 47.41 \\
$(14,1.2,2)$ & 4.14 & 9.65 & 0.02 & 0.08 & 4.98 & 51.61 & 56.08 \\
$(14,1.2,4)$ & 3.27 & 9.34 & 0.02 & 0.08 & 4.99 & 44.20 & 52.17 \\
$(14,1.2,8)$ & 3.18 & 9.02 & 0.02 & 0.08 & 5.05 & 48.38 & 57.63 \\
$(14,1.6,2)$ & 4.10 & 8.96 & 0.02 & 0.08 & 3.80 & 54.23 & 62.52 \\
$(14,1.6,4)$ & 3.31 & 8.10 & 0.02 & 0.08 & 3.81 & 55.13 & 61.38 \\
$(14,1.6,8)$ & 3.16 & 7.86 & 0.03 & 0.08 & 3.80 & 53.06 & 58.63 \\
\hline
\end{tabular}

Table 7: CPU seconds required by the benchmark methods to compute one set of bid prices for the second airline network. The runtimes that are less than 5 milliseconds are indicated as zero. 


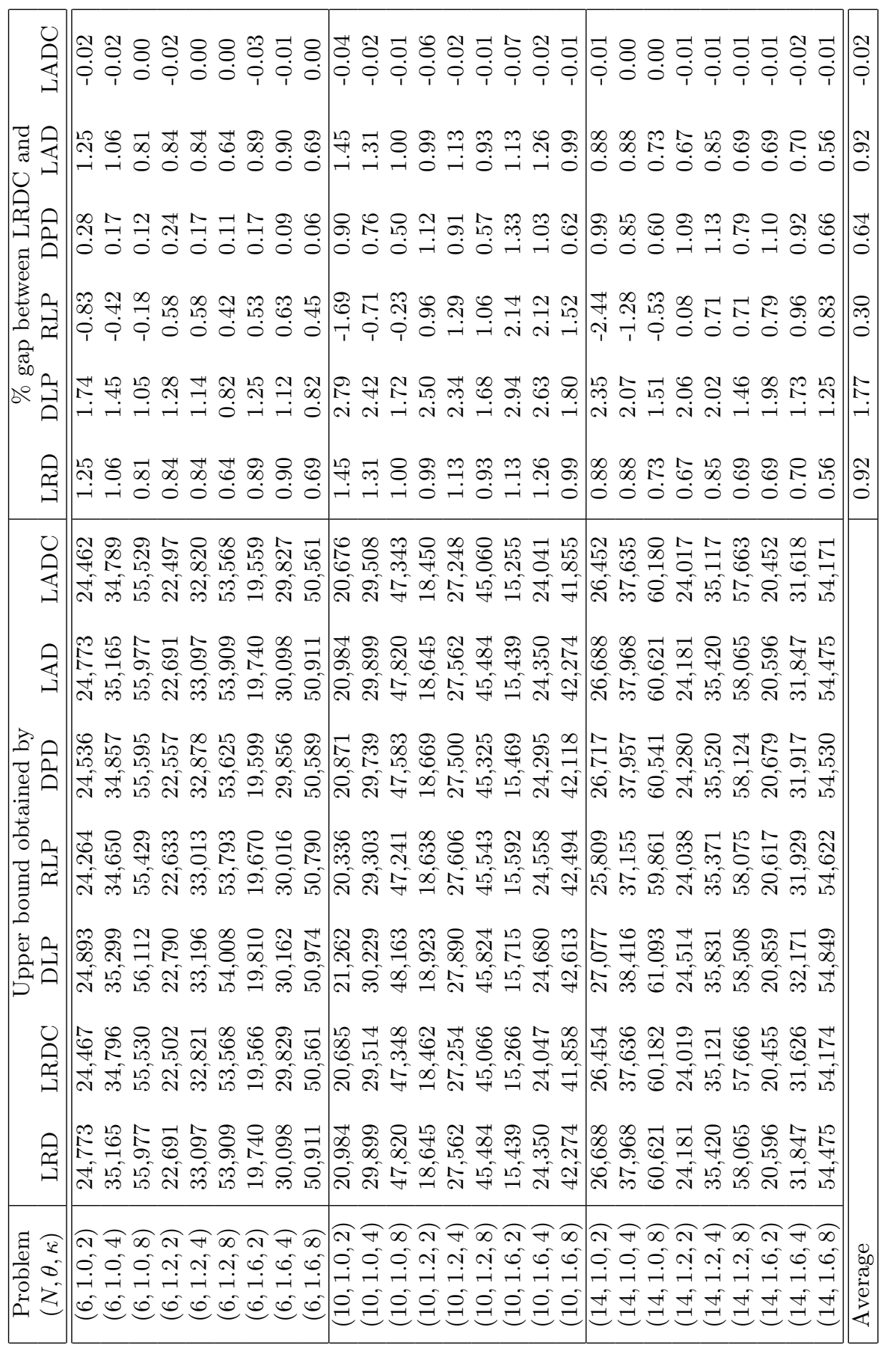

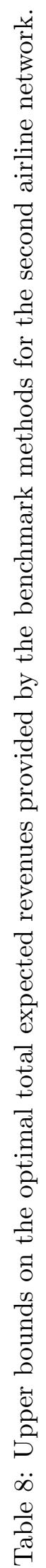

